\title{
Differential diffusion of high-Schmidt-number passive scalars in a turbulent jet
}

\begin{tabular}{|c|c|}
\hline Journal: & Journal of Fluid Mechanics \\
\hline Manuscript ID: & JFM-07-S-0686.R1 \\
\hline mss type: & Standard \\
\hline $\begin{array}{r}\text { Date Submitted by the } \\
\text { Author: }\end{array}$ & $\mathrm{n} / \mathrm{a}$ \\
\hline Complete List of Authors: & $\begin{array}{l}\text { Mydlarski, Laurent; McGill University, Mechanical Engineering } \\
\text { Gaskin, Susan; McGill University, Civil Engineering and Applied } \\
\text { Mechanics } \\
\text { Lavertu, Thomas; GE Global Research Center }\end{array}$ \\
\hline Keyword: & Turbulent mixing < Mixing \\
\hline
\end{tabular}

\section{ScholarONE" \\ Manuscript Central}




\title{
Differential diffusion of
}

\section{high-Schmidt-number passive scalars in a}

\section{turbulent jet}

\author{
By T.M. LAVERTU ${ }^{1}$, L. MYDLARSKI ${ }^{2}$, and S.J. GASKIN ${ }^{3}$ \\ ${ }^{1}$ GE Global Research Center, 1 Research Circle, Niskayuna, NY, 12309, USA \\ ${ }^{2}$ Department of Mechanical Engineering, McGill University \\ ${ }^{3}$ Department of Civil Engineering and Applied Mechanics, McGill University \\ 817 Sherbrooke Street West, Montréal, QC, H3A-2K6, CANADA
}

(Received 29 May 2008)

The separate evolution, or differential diffusion, of high-Schmidt-number passive scalars in a turbulent jet is studied experimentally. The two scalars under consideration are disodium fluorescein $(S c \equiv \nu / D=2000)$ and sulforhodamine $101(S c=5000)$. The objectives of the research are twofold: to determine i) the Reynolds-number-dependence, and ii) the radial distribution of differential diffusion effects in the self-similar region of the jet. Punctual laser-induced fluorescence (LIF) measurements were obtained 50 jet diameters downstream of the nozzle exit for five Reynolds numbers $\left(R e \equiv u_{o} d / \nu=900\right.$, $2100,4300,6700$, and 10600 , where $u_{0}$ is the jet exit velocity, $d$ is the jet diameter, and $\nu$ is the kinematic viscosity) and for radial positions extending from the centreline to the edges of the jet cross-section $(0 \leqslant r / d \leqslant 7.5)$. Statistics of the normalized concentration difference, $Z$, were used to quantify the differential diffusion. The latter were found to slowly decay with increasing Reynolds number, with the RMS of $Z$ scaling as $Z_{r m s} \equiv\left\langle Z^{2}\right\rangle^{1 / 2} \propto R e^{-0.1}$, (or alternatively $\left\langle Z^{2}\right\rangle \propto R e^{-0.2}$ ). Regardless of Reynolds number, differential diffusion effects were found to increase away from the centreline. The increase in differential diffusion effects with radial position, along with their increase with decreasing Reynolds number, support the hypothesis of increased differential diffusion at interfaces between the jet and ambient fluids. Power spectral densities of $Z$ were also studied. These spectra decreased with increasing wavenumber - an observation attributed to the decay of the scalar fluctuations in a turbulent jet. Furthermore, these spectra showed that significant differential diffusion effects persist at scales larger than the Kolmogorov scale, even for moderately high Reynolds numbers. 


\section{Introduction}

The mixing of scalars (e.g., temperature, chemical species, pollutants, etc.) within a turbulent flow is a common occurrence, relevant to a multitude of engineering and environmental applications, including environmental pollutant dispersion, heat transfer, and combustion. The subject, being of both fundamental and practical importance, has been extensively studied. Overviews of this field of research can be found in Sreenivasan (1991), Shraiman \& Siggia (2000), Warhaft (2000), and Dimotakis (2005).

Due to the complexity of turbulent flows, the majority of scalar mixing studies have focused on the evolution of a single scalar. However, within certain flows, more than one scalar is often mixed. Two such examples are the transport of both temperature and salinity in the ocean, and the mixing of two or more reactants in a chemically-reacting flow.

Whenever multiple scalars, with different molecular diffusivities, are transported by a turbulent flow, there exists the possibility that they will evolve separately from one another. If the scalars are initially perfectly correlated and subject to the same initial and boundary conditions, as well as the same velocity field, any differences in their evolution will be exclusively due to their unequal molecular diffusivities. This phenomenon is known as differential diffusion.

Kolmogorov (1941) theory implies that, at high enough Reynolds numbers, differential diffusion effects should be negligible, since they are molecular in origin. Currently, most turbulent mixing models neglect differential diffusion effects by making one of two assumptions. The first is to assume that all the scalars have equal molecular diffusivities, thereby reducing the number of equations to be solved (since each scalar is then governed by the same advection-diffusion equation). The second assumption is to neglect the effects of molecular diffusivity by assuming that the turbulent transport terms are much 
Differential diffusion of high-Schmidt-number passive scalars in a turbulent jet 3 greater than the molecular transport terms. Clearly, the first assumption is incorrect and the second is questionable considering the low to moderate Reynolds numbers that characterize many practical flows. Hence, a thorough understanding of differential diffusion (and its implication for engineering and environmental applications) is necessary.

Differential diffusion effects were first observed by Bilger (1977) when he recast the methane diffusion flame data of Tsuji and Yamaoka (1971), thus providing the motivation for further studies of the subject. Subsequently, Bilger (1981) and Bilger and Dibble (1982) developed theoretical equations to describe and quantify differential diffusion effects in turbulent diffusion flames and non-reacting flows, respectively.

Bilger and Dibble (1982) defined a variable, $z$, to quantify differential diffusion effects based on the differences in mixture fractions of two species. They specified the mixture fraction for a given species $i$ as:

$$
\xi_{i} \equiv \frac{Y_{i}-Y_{i, 2}}{Y_{i, 1}-Y_{i, 2}},
$$

where $Y_{i}$ is the mass fraction of species $i$, and $Y_{i, 1}$ and $Y_{i, 2}$ are the mass fractions of species $i$ in two fluid streams, 1 and 2 . The difference in the mixture fraction of species $(i$ and $j)$, defined as:

$$
z \equiv \xi_{i}-\xi_{j},
$$

was used to quantify the differential diffusion.

Subsequent research studying differential diffusion has been both numerical and experimental in nature. Yeung and Pope (1993) performed the first direct numerical simulations (DNSs) of differential diffusion. They observed differential diffusion effects, characterized by a complete de-correlation of their scalar fields, in homogeneous, isotropic turbulence. Yeung (1996) studied the spectral mechanisms of differential diffusion in sta- 
tionary, isotropic turbulence by means of DNS. When a mean scalar gradient, acting as a source of scalar production, was imposed on the flow, differential diffusion effects were only manifested at the small scales. However, in the absence of imposed mean scalar gradients (i.e., a flow with decaying scalar fields), differential diffusion effects propagated from the small to the large scales. Nilsen and Kosály (1997) also performed a DNS in which they observed a de-correlation of their scalar fields in decaying isotropic turbulence. Yeung and coworkers expanded on earlier DNS studies (i.e., Yeung and Pope, 1993) by performing their simulations at higher Reynolds (Yeung, 1998) and Schmidt numbers (Yeung et al., 2000). The latter study, conducted at Schmidt numbers as large as 4, demonstrated a propagation of scalar incoherency, from small to large scales, that was Schmidt-number-dependent.

A variety of models have also been developed to describe differential diffusion effects. Bilger and Dibble (1982) first used a $k-\epsilon$ model to simulate a jet of hydrogen and propane. At a Reynolds number of 2700 , they observed differential diffusion effects, as they obtained RMS values of $z\left(z_{r m s} \equiv\left\langle z^{2}\right\rangle^{1 / 2}\right)$ greater than 0.004 .

Kerstein and coworkers have used the linear-eddy model to study differential diffusion. Kerstein (1990) simulated a turbulent jet of a hydrogen/propane mixture at Reynolds numbers of 5000 and 20000. Differential diffusion effects were observed at each Reynolds number, with a decrease in $z_{r m s}$ at the higher Reynolds number. Kerstein et al. (1995) defined a normalized concentration difference as:

$$
Z \equiv \frac{C_{A}}{\left\langle C_{A}\right\rangle}-\frac{C_{B}}{\left\langle C_{B}\right\rangle}
$$

The results of their simulations, where the Reynolds number ranged from 100 to 8000, predicted a decay in differential diffusion with Reynolds number, where the variance of $\mathrm{Z}$ scaled as $R e^{-1 / 2}$ (or, alternatively, $Z_{r m s} \propto R e^{-1 / 4}$ ). 
Differential diffusion of high-Schmidt-number passive scalars in a turbulent jet 5 The conditional moment closure (CMC) has also been used to study differential diffusion in non-reacting (Kronenburg and Bilger, 1997) and reacting flows (Kronenburg and Bilger, 2001a and 2001b). In each study, the predictions of the CMC model were compared to data from DNSs.

Other attempts to model differential diffusion include the work of Pitsch and Peters (1998) and Pitsch (2000), who employed flamelet formulations to calculate differential diffusion effects in combustion applications, and that of Chen and Chang (1998), who used a stochastic mixing model for the prediction of differential diffusion in non-reacting jets. A Lagrangian spectral relaxation model was developed for the study of differential diffusion in forced, homogeneous, isotropic turbulence (Fox, 1999). It predicted the variance of the scalar difference to scale as $R e^{-0.3}$ (or $Z_{r m s} \propto R e^{-0.15}$ ), a weaker decay than that predicted by Kerstein et al. (1995). Eddy-damped quasi-normal Markovian (EDQNM) theory was also utilized to study differential diffusion (Ulitsky, Vaithianathan, and Collins, 2002). The results were consistent with the DNS of Yeung (1996), indicating that differential diffusion is a molecular phenomenon and mean scalar gradients act as a source of coherency for the large scales, preventing the propagation of differential diffusion effects to these scales.

Experimental studies have focused on determining differential diffusion effects in both reacting and non-reacting flows. Drake, Pitz, and Lapp (1986) observed differential diffusion effects in hydrogen/air jet diffusion flames persisting up to Reynolds numbers of 8500. Similarly, Masri, Dibble, and Barlow (1992) found differential diffusion effects to be insignificant at high Reynolds numbers (up to 30000) in their study of hydrogen/carbon dioxide turbulent flames. However, they noted that significant differential diffusion effects may still have been present in the minor species, for which they were unable to obtain 
results. Conversely, Smith et al. (1995b) observed differential diffusion effects up to Re $=30000$ in their jet flames consisting of a hydrogen/carbon dioxide mixture.

In reacting flows, differential diffusion effects appear to be most prominent on the fuel rich side of the flame. Smith et al. (1995b), Meier et al. (1996), and Bergmann et al. (1998) all report this phenomenon in their studies of jet diffusion flames.

Non-reacting experimental studies have been conducted in liquid and gas-phase flows. There are two main differences between these two types of flows. Firstly, gas-phase flows typically have Schmidt numbers of $O(1)$ (and hence the scalar dissipative scales are of the same order as the Kolmogorov scale). Secondly, inertial effects are often present in gasphase experiments. Although important, their presence makes it difficult to isolate the effects of differential diffusion from those of the density differences generally present in multi-component gas flows. Liquid-phase studies typically have Schmidt numbers $O\left(10^{3}\right)$ (and scalar fields with fluctuations extending to scales much smaller than the Kolmogorov microscale). Furthermore, these studies can be relatively easily conducted in the absence of inertial effects (due to the increased density of liquids as compared to gases), facilitating the isolation of differential diffusion effects.

Dibble and Long (2005) reported on a non-reacting gas-phase study, proposed by Bilger and Dibble (1982) and conducted in 1989. Differential diffusion effects were observed in jets containing mixtures of Freon and hydrogen for Reynolds numbers up to 20000. Long, Starner, and Bilger (1993) observed differences in structures of their tracers, indicating the presence of differential diffusion, from images of jets with $R e=3300$ and 10000 . Smith et al. (1995a) conducted experiments in jets of hydrogen/carbon dioxide for Reynolds numbers as high as 64000 . They found differential diffusion effects to be present at all Reynolds numbers, although, $z_{r m s}$ decreased with increasing Reynolds number. Brownell and $\mathrm{Su}(2004,2008)$ reported on experiments in a jet containing a mixture of propane 
Differential diffusion of high-Schmidt-number passive scalars in a turbulent jet 7 and helium, for Re $=1000-3000$. They observed a decrease in differential diffusion effects with downstream distance and increasing Reynolds number, off-axis peaks of the differential diffusion intensity, and monotonically decaying spectra of the differential diffusion variable.

Saylor and Sreenivasan (1998) conducted a liquid-phase study to determine differential diffusion effects in a low-Reynolds-number turbulent water jet $(\mathrm{Re}=430)$. Employing two Schmidt number ratios (4 and 18), they observed differential diffusion effects to be present. As can be expected, these effects were more prominent when using the higher Schmidt number ratio, for which $Z_{r m s}$ increased from 0.11 to 0.31 .

The objective of the present work is to quantify the differential diffusion of highSchmidt-number passive scalars in a flow at moderate Reynolds numbers. This is accomplished by studying the evolution of two passive scalars (fluorescent dyes mixed in water) in an axisymmetric turbulent water jet issuing into a quiescent background. In particular, the Reynolds number dependence and radial distribution of differential diffusion effects are sought. A normalized concentration difference is defined as:

$$
Z \equiv \frac{C_{1}}{\left\langle C_{1}\right\rangle}-\frac{C_{2}}{\left\langle C_{2}\right\rangle},
$$

where $C_{1}$ and $C_{2}$ are the instantaneous concentrations of species 1 (disodium fluorescein, $\mathrm{Sc}=2000$ ) and 2 (sulforhodamine 101, Sc=5000), respectively $\dagger$. Each scalar is normalized by its average concentration at that point in the flow field, similar to the method of Saylor and Sreenivasan (1998). The statistics of $\mathrm{Z}$ will be studied to elucidate our understanding of differential diffusion.

The remainder of this paper is organized as follows. A detailed description of the apparatus is provided in $\S 2$. The experimental conditions and flow validation are discussed 
in $\S 3$. Results pertaining to differential diffusion are presented in $\S 4$. These include time series, statistical moments, spectra, probability density functions (PDFs) and conditional expectations of the normalized concentration difference, $Z$. As the results are presented, comparisons with previous work will be made. A discussion of the results will be presented in $\S 5$. It will centre on two main points, i) a physical mechanism resulting in increased differential diffusion, and ii) the form of the spectrum of the normalized concentration difference. The conclusions of the current work will be presented in $\S 6$. Lastly, an uncertainty analysis, in which the sources of error are discussed and quantified, is presented in the Appendix.

\section{Apparatus}

Instantaneous species concentrations were obtained by punctual laser-induced fluorescence (LIF) measurements. A thorough description of the experimental setup is provided below and divided into three subsections. The first describes the jet components (§2.1). It is followed by a review of the optical and signal detection equipment (§2.2). Finally, a description of the data acquisition and signal processing is provided (§2.3). The interested reader is also referred to Lavertu (2006).

\subsection{Jet setup}

The flow under consideration was an axisymmetric turbulent water jet issuing into a quiescent background. The background fluid was contained in a large glass tank $(6 \mathrm{~m}$ long by $1.5 \mathrm{~m}$ wide by $1 \mathrm{~m}$ high) with the top open to the ambient. A minimum of 3 hours were allowed to pass (after filling the tank) before the start of an experiment.

Figure 1 depicts the supply reservoir, constant head reservoir, and jet components. It was designed to ensure that the flow exiting the jet nozzle was constant throughout each 
Differential diffusion of high-Schmidt-number passive scalars in a turbulent jet 9 experiment, while allowing the Reynolds number to be easily varied from one experiment to another.

The supply reservoir, a $35 \ell$ cylindrical glass container, served three purposes. Firstly, it was used to thoroughly mix the fluorescent dyes before conducting an experiment. Secondly, it served as a reservoir in each test - the dyed fluid was pumped from it up to the constant head reservoir, via a 1/3 HP pump (Franklin Electric 1113910404). Thirdly, the supply reservoir also acted as an overflow reservoir for the excess dyed fluid that was pumped to the constant head reservoir. (To maintain a steady level in the constant head reservoir, the flowrate into the constant head reservoir was set to be slightly greater than that through the turbulent jet.)

The constant head reservoir (a $12 \ell$ spherical glass container located $2 \mathrm{~m}$ above the jet nozzle) created the pressure difference used to drive the fluid through the jet. It ensured that the jet flowrate, and therefore Reynolds number, remained constant during each experiment. Upon exiting the constant head reservoir, the flow was first directed through a ball valve and then a flowmeter (OMEGA FL 50002), connected in series. These devices were used in tandem to control the jet flowrate (i.e., Reynolds number) to within $6 \%$. The flowrate was varied from $0.7 \ell \mathrm{min}^{-1}$ (corresponding to $\mathrm{Re}=900$ with a $1.6 \mathrm{~mm}$ diameter jet) to $4.0 \ell \mathrm{min}^{-1}$ (corresponding to Re $=10600$ with an $8 \mathrm{~mm}$ diameter jet). After the flowmeter, the fluid passed through a solenoid valve, which was used to control whether or not there was flow through the turbulent jet apparatus.

Upon exiting the solenoid valve, the flow was contracted into $8 \mathrm{~mm}$ inner diameter copper tubing. This tubing extended vertically down $50 \mathrm{~cm}$ into the tank, at which point there was a $90^{\circ}$ bend. After the bend, the tubing extended horizontally for another 12 $\mathrm{cm}$. The fluid exited the tubing and entered into the quiescent, ambient fluid when an 8 mm diameter jet was required. When a smaller jet diameter was required, the flow out 
of the $8 \mathrm{~mm}$ diameter copper tubing was contracted and entered into one of two 50.8 mm long stainless steel dispensing needles whose outlets served as the jet exit. A 7 gauge needle (McMaster-Carr 6710A13) provided a $3.81 \mathrm{~mm}$ diameter jet, whereas a 14 gauge needle (McMaster-Carr 6710A21) resulted in a $1.6 \mathrm{~mm}$ diameter jet. Given the different assemblies used to achieve the three different jet diameters, there were slight differences in the initial jet conditions / exit profiles. However, it has been shown (Antonia and Zhao, 2001) that the effect of the exit velocity profile in a round jet is "forgotten" for $x / D \gtrsim 30$, where the spectra of the axial and radial velocity fluctuations collapse over all scales. Similar conclusions were drawn for the velocity spectra, profiles of Reynolds shear stress and the autocorrelations of axial and radial velocities in Xu and Antonia (2002a). Furthermore, $\mathrm{Xu}$ and Antonia (2002b) obtained similar results for the PDF, skewness and kurtosis of the temperature fluctuations, the correlation coefficient of longitudinal velocity and temperature, and the autocorrelation of temperature fluctuations in a heated jet with top-hat and fully-developed velocity profiles. Lastly, in a study that compared turbulent round jets issuing from a straight pipe to those issuing only a few jet diameters downstream of a $90^{\circ}$ bend, Ferdman et al. (2000) showed that the far-field velocity statistics of a round jet with i) an (axisymmetric) fully-developed velocity profile, and ii) an asymmetric initial profile, were experimentally indistinguishable in the far-field. In each instance, they found that i) the mean velocities were Gaussian and self-similar by 15 jet diameters downstream of the nozzle, and ii) the turbulence intensities were self-similar by 40 jet diameters. Furthermore, Ferdman et al. (2000) also observed the differences in i) the mean centreline axial velocities, and ii) the normalized rms velocities arising from the different initial conditions to be negligible in the far-field. Because all of the measurements were made at $x / D=50$, the slight differences in jet initial conditions should not affect our results. This was confirmed by measurements herein that were 
Differential diffusion of high-Schmidt-number passive scalars in a turbulent jet 11 repeated at the same Reynolds number, but with different jet exit diameters (see $§ 3.1$ / Table 1 for the different combinations).

The copper tubing was mounted on two traversing mechanisms (Velmex A15 and A25 series), positioned one on top of the other, above the free surface of the water. These enabled the jet to be positioned to within $\pm 0.05 \mathrm{~mm}$ in both the horizontal and vertical directions. To vary the radial measurement location within the jet, the latter's position within the tank was changed, as it was impractical to continually move the optical equipment (described in the next subsection). Hence, the (absolute) measurement location remained fixed for all experiments.

The fluid entering the tank consisted of a dilute mixture of two fluorescent dyes. Dilute concentrations served multiple useful purposes. Firstly, they ensured that the emitted fluorescence was linearly related to the species concentration. Secondly, they aided in preventing inertial (e.g., buoyancy), trapping/absorption, and/or laser attenuation effects. See the uncertainty analysis in Appendix A for the details of the tests performed to i) quantify these and other effects, and ii) ensure that they were indeed negligible.

Disodium fluorescein (Fisher Scientific) and sulforhodamine 101 (Biotium) were employed because of their differing molecular diffusivities and fluorescence characteristics. The dye concentrations employed herein were $2 \times 10^{-7} \mathrm{~mol} / \ell$ and $6 \times 10^{-7} \mathrm{~mol} / \ell$, respectively. When diluted in water $\left(\nu \sim 10^{-6} \mathrm{~m} \mathrm{~s}^{-2}\right)$, the Schmidt number of disodium fluorescein is 2000 (Ware et al. 1983) whereas that of sulforhodamine 101 is 5000 (Saylor 1993). The fluorescence characteristics of these dyes suit the present experiments. Both dyes can be excited by the same excitation source, as evidenced by the overlap in their excitation spectra as shown in figure 2(a). Upon detection, they can be distinguished from each other since they have differing emission spectra. (See figure 2(b).) The dis- 
odium fluorescein spectra in figure 2 were measured by Saylor and Sreenivasan (1998), while those of sulforhodamine 101 were provided by the manufacturer.

\subsection{Optical setup}

The optical setup, depicted in figure 3, consisted of both laser-focusing and signaldetection equipment. A description of this equipment is given below.

A laser beam issued from an argon-ion laser (Coherent Innova 90) to irradiate the fluorescent dyes at the measurement location. The laser was operated in single-line mode, with a $514.5 \mathrm{~nm}$ beam exiting the $3.97 \mathrm{~mm}$ aperture at a power of $1.5 \mathrm{~W}$. The 514.5 $\mathrm{nm}$ line was selected since it fell within the excitation spectra of both fluorescent dyes (see figure 2). It was also operated in light regulation mode, thus providing a long-term power stability of $\pm 0.5 \%$.

When a fluorescent dye is continuously irradiated by a laser, the signal will eventually degrade due to photobleaching and/or thermal blooming effects (i.e., Sahar and Treves 1977, Koochesfahani 1984, Benson et al. 1985, Saylor 1995, Crimaldi 1997, Wang and Fiedler 2000). Therefore, upon exiting the laser, the beam was first passed through a high-speed optical chopper (Scitec 310CD). This device served to pulse the beam and therefore prevent photobleaching and/or thermal blooming effects. (See Appendix A for the calculation of the photobleaching and thermal-blooming time scales.) It was operated with two five-slot discs - one on top of the other - that were offset at an angle. Simultaneous adjustment of the angle between the two discs and the chopper's (angular) speed allowed independent control of the duration of the open and closed segments of the pulse (i.e., the period and duty cycle of the pulse could be independently varied). For each experiment, the duration of the open segment was always $150 \mu \mathrm{s}$, whereas the duration of the closed segment varied, depending on the velocity of the jet for the experiment under consideration. The latter therefore ultimately dictated the chopping frequency and 
Differential diffusion of high-Schmidt-number passive scalars in a turbulent jet 13 was selected to ensure that the fluid parcels passing through the measurement volume were not exposed to significant photobleaching by remaining in the measurement volume for excessively long periods. (See Appendix A for the details.) The resulting chopping frequencies varied from 651 to $1606 \mathrm{~Hz}$ (see $\S 3.1$ ).

To focus the (chopped) laser beam to a small diameter at the measurement location, it was first directed through a $3 \times$ beam expander (Melles Griot 091BZ001) before being focused by a $1 \mathrm{~m}$ focal length $(f)$ plano-convex lens (Melles Griot 011PX347). These components served to focus the laser beam down to a waist diameter $\left(d_{0}\right)$ of approximately $100 \mu \mathrm{m}$, as determined from:

$$
d_{0}=\frac{2.44 f \lambda}{D}
$$

where $\lambda$ is the wavelength of the incident beam and $D$ is its diameter as it enters the focusing optic (Ready 1978).

The fluorescence emitted from the measurement location was focused onto the signal detection devices (two photomultiplier tubes or PMTs) by the signal detection optics. Most of these optics were mounted on a $2 \mathrm{~m}$ optical rail (Melles Griot 070RN011) positioned perpendicular to the axis of the laser beam, whereas the rest were secured to a second, smaller $(0.25 \mathrm{~m})$ optical rail (Melles Griot 070RN001), perpendicular to the longer one.

The first optical component along the $2 \mathrm{~m}$ optical rail was a $0.5 \mathrm{~m}$ focal length achromatic lens (Melles Griot 011A0346). It was positioned in between the laser beam waist and a $100 \mu \mathrm{m}$ pinhole (Melles Griot 04PPM015 - also secured on the $2 \mathrm{~m}$ optical rail), $1 \mathrm{~m}$ from each component. The purpose of the pinhole was to limit the fluorescence detected by the PMTs to that emitted from a $100 \mu \mathrm{m}$ length along the axis of the beam (at the measurement location / beam waist). This was possible because of the one-to-one 
relationship between the object distance and the image distance, as can be seen from the lens and magnification equations (Jenkins and White 1957, Rossi 1957):

$$
\begin{gathered}
\frac{1}{f}=\frac{1}{s}+\frac{1}{s^{\prime \prime}} \\
M=\frac{s^{\prime \prime}}{s}
\end{gathered}
$$

where $s$ is the object distance, $s^{\prime \prime}$ is the image distance, and $\mathrm{M}$ is the magnification. Thus, the emitted fluorescence signal that passed through the pinhole (and reached the PMTs) originated from a $100 \mu \mathrm{m}$ length along the axis of the laser beam. The pinhole and achromat (at their respective positions) ensured that the resolution of the experiments was a maximum of $100 \mu \mathrm{m}$ in each direction.

After passing through the pinhole, the emitted fluorescence needed to be simultaneously focused onto the two PMTs. To this end, a $60 \mathrm{~mm}$ focal length achromat (Melles Griot 011A0079) was secured $120 \mathrm{~mm}$ past the pinhole, on the $2 \mathrm{~m}$ optical rail. Next, a 50/50 plate beamsplitter (Melles Griot 03BTF023), located $40 \mathrm{~mm}$ from the $60 \mathrm{~mm}$ focal length achromat on the optical rail, was utilized to split the signal and direct it towards both PMTs.

Two PMTs (Hamamatsu R928), located $80 \mathrm{~mm}$ from the beamsplitter, were employed to detect the emitted fluorescence. One of the PMTs was secured on the $0.25 \mathrm{~m}$ optical rail, positioned perpendicular to the longer optical rail at the beamsplitter location. The other PMT was positioned on the $2 \mathrm{~m}$ optical rail. Each PMT, housed in an aluminum enclosure, had a different optical filter placed in front of it. A bandpass filter (Andover Corporation 550FS40-25) with a $40 \mathrm{~nm}$ bandwidth centred at $550 \mathrm{~nm}$ was placed in front of the PMT on the $2 \mathrm{~m}$ optical rail, thus effectively permitting only the fluorescence emitted by disodium fluorescein to reach this PMT (designated as the disodium fluorescein 
Differential diffusion of high-Schmidt-number passive scalars in a turbulent jet 15 PMT). A $645 \mathrm{~nm}$ color glass cut-off filter (Melles Griot 03FCG505) was placed in front of the PMT on the $0.25 \mathrm{~m}$ optical rail, thus effectively permitting only the emitted fluorescence from sulforhodamine 101 to reach this PMT (designated as the sulforhodamine 101 PMT). Lastly, the PMTs were powered by an Agilent triple output DC power supply (Model E3630A) with $0.01 \%$ load regulation.

\subsection{Data acquisition and signal processing}

The output of each PMT was a current that was passed through a cable towards a unitygain operational amplifier (National Semiconductor LM 747), with a $100 \mathrm{k} \Omega$ resistor placed in parallel with the cable. The resistor was used to convert the output current to a voltage, while also providing gain. The frequency response of the PMTs was lowered due to the resistors, and estimated to be $5900 \mathrm{~Hz} \dagger$. This nevertheless granted a sufficiently high frequency response, while providing enough gain to detect the fluorescence signal. The unity-gain operational amplifiers reduced the current to the data acquisition board while maintaining the same voltage. This reduction in current was necessary to prevent crosstalk between the channels of the data acquisition board.

The output voltages of each PMT, as well as one from the optical chopper, were sent to a 12-bit A/D board (National Instruments PC-MIO-16E4), operating with a $\pm 5 \mathrm{~V}$ sampling window. Acquisition of an output signal from the optical chopper indicated when the laser passed through it and when the beam was blocked.

Each channel was sampled at $83.3 \mathrm{kHz}$. During each experiment, 40 blocks of data were sampled, with each block containing either $2^{18}$ or $2^{19}$ samples per channel (depending on the Reynolds number and jet diameter). Given that each block contained data from

$\dagger$ The frequency response of the PMTs is calculated from $f_{c}=1 /\left(2 \pi R C_{j}\right)$ (Hamamatsu 2006), where $f_{c}$ is the cut-off frequency of the photodetector, $R$ is the total resistance, and $C_{j}$ is the characteristic capacitance of the circuit. The latter was estimated to be $269 \mathrm{pF}$, as each PMT had a nominal capacitance of $20 \mathrm{pF}$, while the capacitance of the cable was nominally $83 \mathrm{pF} / \mathrm{m}$ and $3 \mathrm{~m}$ of cable were used. 
both i) intervals for which the laser beam was blocked by the chopper blades, and ii) intervals for which it passed through the chopper, post-processing was necessary. During this step, data from each (unblocked) laser pulse was averaged to constitute one data point, similar to the method of Saylor and Sreenivasan (1998). However, not all of this data was used in the average, given that these included data points for which the laser beam was partially blocked as the slotted region of the disc in the optical chopper passed through the beam. Therefore, the recorded data points corresponding to times for which the laser beam was partially blocked were also discarded (in addition to ones where the beam was completely blocked). Averaging of the data points from one laser pulse only occurred for data points corresponding to times when the beam fully passed through the slots in the optical chopper. The duration of these times was approximately $100 \mu \mathrm{s}$, and the final sampling frequency was therefore the chopping frequency. The post-processed data were then grouped into blocks of $2^{12}$ samples (per channel), to lend themselves to spectral analysis.

Even though the individual fluorescent dyes were operated within their respective linear regions, an extensive analysis of different calibration procedures was implemented to determine the best method to relate the PMT output voltages to species concentrations. This was motivated by the fact that two dyes with different emission spectra were being excited, which could lead to the detection of the fluorescence of disodium fluorescein by the PMT measuring the fluorescence of sulforhodamine 101 (and vice versa) - so called "optical crosstalk." The latter is calculated in the uncertainty analysis (Appendix A) and found to be less than $1 \%$. Nevertheless, an extensive analysis of different calibration procedures was implemented to determine the best method to relate the PMT output voltages to species concentrations. Five different relationships $\left(C_{\alpha}=f\left(V_{1}, V_{2}\right)\right.$, where $C_{\alpha}$ is the is the species concentration and $V_{1}$ and $V_{2}$ are the voltage outputs of the 
Differential diffusion of high-Schmidt-number passive scalars in a turbulent jet

two PMTs) were studied and compared with the results obtained in jets with only one of the dyes- see Lavertu (2006) for the details. Ultimately, it was confirmed that the most accurate calibration was one in which the output voltage of each PMT was linearly proportional to its respective scalar concentration $\left(C_{\alpha}=K_{\alpha} V_{\alpha}\right)$ - a further confirmation of the absence of optical crosstalk. Given that all data were normalized by the mean concentration $(C /\langle C\rangle)$, it was not necessary to determine the constant of proportionality $\left(K_{\alpha}\right)$, as $\frac{C}{\langle C\rangle}=\frac{V}{\langle V\rangle}$.

A Wiener filter (Wiener 1949) was finally applied to the processed data to eliminate noise introduced by the amplification process in the PMTs. The technique was similar to that implemented by Dowling (1988) and Miller (1991) (see also Dowling, Lang and Dimotakis 1989; Dowling and Dimotakis, 1990; Miller and Dimotakis 1991a; Miller and Dimotakis 1991b; Miller and Dimotakis, 1996) and followed the steps as outlined by Press et al. (1992). Its application significantly increased the signal-to-noise ratio at high frequencies, as evidenced in the disodium fluorescein spectra (before and after filtering) plotted in figure 4 .

\section{Experimental conditions and flow validation}

This section begins by presenting the experimental conditions, enabling the quantification of the (spatial and temporal) resolution of the measurements. A discussion in which single-dye statistics from the present work are compared with previous studies of the scalar mixing in turbulent jets (to validate the measurements) follows.

\subsection{Experimental conditions}

Experiments were conducted at five different jet Reynolds numbers $(R e=900,2100$, 4300, 6700, and 10600), using three jet diameters $(d=1.6,3.81$, and $8 \mathrm{~mm})$. At each Reynolds number, the jet was traversed radially from the centreline towards the edges, 
with data acquisition occurring at eight locations $(r / d=0,0.625,1.25,2.5,3.75,5,6.25$, and 7.5). Measurements were obtained at a downstream distance of $x / d=50$ for all Reynolds numbers, jet diameters, and radial positions.

Length and time scales were calculated at each combination of Reynolds number and jet diameter to determine the spatial and temporal resolution of the experiments. Since the flow consisted of high-Schmidt-number scalars $(S c \gg 1)$, scalar fluctuations were dissipated at the Batchelor scale (Batchelor 1959):

$$
\eta_{b} \equiv{\frac{D^{2} \nu^{1 / 4}}{\epsilon}}^{1 / 4} \eta c^{-1 / 2}
$$

where $\epsilon$ is the dissipation rate of turbulent kinetic energy and $\eta\left(\equiv\left(\nu^{3} / \epsilon\right)^{1 / 4}\right)$ is the Kolmogorov microscale.

Given that only concentration was measured in the present work, previous experimental results were used to determine $\epsilon$ in the jet. The dissipation rate of turbulent kinetic energy was inferred from the correlation of Friehe, Van Atta and Gibson (1971):

$$
\epsilon=48\left(\frac{u_{0}^{3}}{d}\right)\left(\frac{x-x_{0}}{d}\right)^{-4}
$$

where $u_{0}$ is the jet exit velocity and $x_{0}$ is the virtual origin (assumed to be zero in the present work).

Table 1 lists the Kolmogorov and Batchelor scales of the individual scalars at the centreline of the jet, for all combinations of Reynolds number and jet diameter. Clearly, the Batchelor scale is not resolved at any Reynolds number, whereas the Kolmogorov microscale is resolved for all Reynolds numberst. Though differential diffusion has its

$\dagger$ Even though the calculations indicate that the Kolmogorov microscale is under-resolved by $2 \%$ for $\operatorname{Re}=2100$ with the $1.6 \mathrm{~mm}$ jet, it was nevertheless resolved at this Reynolds number using the $3.81 \mathrm{~mm}$ diameter jet. 
Differential diffusion of high-Schmidt-number passive scalars in a turbulent jet

origins at the smallest scales, its effects will be observable at scales larger than $\eta$ (as will be clearly seen in $\S 4$ ), which are the subject of the present work.

The temporal resolution is determined by the sampling frequency $\left(f_{s}\right)$. As previously mentioned in $\S 2.2$, the latter was set by the optical chopper and chosen to minimize photobleaching and thermal blooming effects. (See Appendix A for the details.) Consequently, the chopping frequency was selected to ensure that a parcel of fluid remained in the $(100 \mu \mathrm{m}$ wide) measurement volume for a limited amount of time (ideally less than $150 \mu \mathrm{s})$, thus minimizing any photobleaching or thermal blooming effects. This calculation required an estimate of the centreline velocity $\left(U_{c l}\right)$. The results of Chen and Rodi (1980):

$$
U_{c l}=6.2 u_{0}\left(\frac{x-x_{0}}{d}\right)^{-1}
$$

were used to this end. The temporal resolution could then be estimated by comparing $f_{s}$ with the Kolmogorov frequency $\left(f_{\eta}\right)$, which is given by:

$$
f_{\eta}=\frac{U_{c l}}{2 \pi \eta}
$$

A comparison of $f_{s}$ and $f_{\eta}$, for each combination of Reynolds number and jet diameter, is provided in table 2. In each instance, the sampling frequency is significantly greater than the Kolmogorov frequency. Hence, at each Reynolds number, the Kolmogorov scale is both spatially and temporally resolved.

\subsection{Flow validation}

The purpose of this section is to compare the present measurements to those from previous studies (of concentration of a single species). Given that the concentration of two species are simultaneously measured in the present work, some measurements with only 
one species present (i.e., disodium fluorescein or sulforhodamine 101) were also made to ensure that the presence of one species did not affect the measurements of the other.

Figure 5 plots the radial profile of the average concentration of each scalar (normalized by its respective centreline value) for $\mathrm{Re}=10600$. Also shown in this figure is the average helium concentration profile as measured by Panchapakesan and Lumley (1993) in their turbulent jet. As expected, the mean concentration decreases with radial position and agrees well with the results of Panchapakesan and Lumley.

We proceed to consider RMS concentrations. However, before doing so, it is worth noting that the normalized RMS concentration fluctuation of a given scalar, $c_{r m s} /\langle C\rangle$, can be determined from the power spectrum of concentration fluctuations:

$$
\frac{c_{r m s}}{\langle C\rangle}=\left[\frac{\left\langle c^{2}\right\rangle}{\langle C\rangle^{2}}\right]^{1 / 2}=\left[\frac{1}{\langle C\rangle^{2}} \int_{0}^{\infty} E_{c}(f) d f\right]^{1 / 2} .
$$

Given that i) the above integral requires that the entire concentration power spectrum be measured, and ii) the Batchelor scale was not resolved in the present experiments, we consider a surrogate of the normalized RMS concentration fluctuation, given by the following:

$$
\frac{c_{r m s, \text { measured }}}{\langle C\rangle}=\left[\frac{\left\langle c^{2}\right\rangle_{\text {measured }}}{\langle C\rangle^{2}}\right]^{1 / 2}=\left[\frac{1}{\langle C\rangle^{2}} \int_{0}^{f_{\eta}} E_{c}(f) d f\right]^{1 / 2}
$$

Were $c_{r m s}$ to be calculated directly from the time series, it could be affected by the differing spatial resolutions from experiment to experiment. $c_{r m s, \text { measured }} /\langle C\rangle$ provides a surrogate for the normalized RMS concentration fluctuation that is consistent for each experiment, independent of the spatial resolution. Note that the values of $c_{r m s, m e a s u r e d}$ presented herein will underestimate the true $c_{r m s}$. That being said, this difference should be small as the majority of the contributions to $c_{r m s}$ are from the large scales, which are entirely resolved. 
Differential diffusion of high-Schmidt-number passive scalars in a turbulent jet 21

Figure 6 plots the RMS concentration of each scalar, as measured at the jet centreline, as a function of Reynolds number. The results for the two individual scalar concentrations are consistent with both i) each other, and ii) those of disodium fluorescein (resolved down to the Batchelor scale) obtained by Miller (1991) and Miller and Dimotakis (1991b).

Figure 7 plots the radial profile of normalized RMS scalar concentration fluctuations for Re $=10600$. This profile, along with that of the helium jet of Panchapakesan and Lumley (1993), is shown. The shape of these profiles - both exhibiting an off-axis peak - is similar. However, the magnitude of the RMS scalar concentration fluctuations is greater in the present high-Schmidt-number (liquid-phase) study than in the low-Schmidt-number (gas-phase) study of Panchapakesan and Lumley (1993). This difference is expected, as RMS scalar fluctuations have previously been observed to be Schmidt/Prandtl number dependent, taking on larger values in high-Schmidt-number, liquid-phase flows (e.g., Miller 1991, Miller and Dimotakis 1991b) than in low-Schmidt-number, gas-phase flows (e.g., Dowling and Dimotakis 1990). The present results would ideally be compared to RMS concentration profiles in high-Schmidt-number flows. However, none were found in the literature and the present results could only be compared to those obtained in a gas-phase flow, where the Schmidt number was significantly lower.

The effect of one fluorescent dye on the other is examined by comparing concentration power spectra of one species when measured i) alone in a jet, and ii) in a jet containing both dyes. Such a comparison is performed in figures 8a) and b). The difference in the normalized RMS concentration of disodium fluorescein (calculated from the two spectra in figure 8 a) by means of equation (3.6)) is $1 \%$. The difference in the normalized RMS concentration of sulforhodamine 101 (calculated from the two spectra in figure 8b) by means of the same equation) is $0.1 \%$. Consequently, the above results serve to indicate that the concentrations of both dyes are accurately measured and that the influence of 
one dye on the other is negligible. Lastly, the spectra of each dye (measured in the same realization of a turbulent jet containing both dyes) are plotted together in figure 8c). (In other words, the spectra denoted by dashed lines in figure 8a) and b) are re-plotted in figure 8c).) They are effectively identical.

\section{Results}

The results presented herein focus on statistics of the normalized concentration difference, $Z$. They are divided into sections which present time series (§4.1), RMS fluctuations (§4.2), PDFs (§4.3), and conditional expectations (§4.4) of the normalized concentration difference.

\subsection{Time series}

Insight into differential diffusion effects can be obtained by studying time series of individual normalized scalar concentrations and their respective normalized concentration difference. When differential diffusion is present, $Z$ will be non-zero.

Individual normalized scalar concentration time series (measured at the jet centreline) are plotted in figure 9 for $\operatorname{Re}=900$ and $\operatorname{Re}=10600$. The scalar time series appear to be well correlated, especially at the large scales. However, there are clear differences in the small-scale structure of the two time series for both Reynolds numbers. These small-scale differences confirm the presence of differential diffusion.

A more precise depiction of the effects of differential diffusion is obtained by examining time series of $Z$ - see figure 10 . The time series of $Z$ depicted in figure 10 correspond to the individual scalar time series in figure 9. Differential diffusion is clearly present at each Reynolds number, as $Z$ is non-zero more often than not. Furthermore, the magnitude of $Z$ is typically greater at the lower Reynolds number, consistent with a decrease in differential diffusion with increasing Reynolds number. 
Differential diffusion of high-Schmidt-number passive scalars in a turbulent jet 23 The time series in figure 10 also provide information on the scales at which differential diffusion effects persist. For both Reynolds numbers, there are instances for which $Z$ remains non-zero for times greater than the Kolmogorov timescale, $\tau\left(\equiv(\nu / \epsilon)^{1 / 2}\right)$, indicating the presence of differential diffusion at these (and larger) scales. Saylor and Sreenivasan (1998) also obtained time series of $Z$ at the centreline of a low-Reynolds-number $(\mathrm{Re}=430)$ turbulent jet. They too observed instances in which $Z$ remained non-zero for times greater than the Kolmogorov timescale. They made such observations for Schmidt numbers similar to those employed herein $\left(S c_{1}=1200\right.$ and $\left.S c_{2}=4400\right)$, as well as for scalars with a larger Schmidt number difference $\left(S c_{1}=4400\right.$ and $\left.S c_{2}=77000\right)$. Consistent with theoretical expectations, they found more occurrences in which $Z$ remained non-zero for times greater than the Kolmogorov timescale when using scalars with the larger Schmidt number difference.

\subsection{RMS measurements of the normalized concentration difference}

The intensity of differential diffusion effects can be quantified by the RMS value of the normalized concentration difference, $Z_{r m s}$. The physical implications of this quantity are the subject of the current section. However, before proceeding to a presentation of the results, we discuss the methodology employed herein to determine consistent values of $Z_{r m s}$ given the spatial resolution of the current experiments, which varies and lies in between the Kolmogorov and the Batchelor scales.

\subsubsection{Methodology for the calculation of $Z_{r m s}$}

The spectrum of $Z, E_{Z}\left(\kappa_{1}\right)$ (where $\kappa_{1}$ is the longitudinal wavenumber), contains the information pertaining to the intensity of the fluctuations in $Z$ at different scales $\dagger$. Consequently, $Z_{r m s}$ can be determined from $E_{Z}$ by integrating the latter over all wavenumbers:

$\dagger$ The nature of $E_{Z}$ will be discussed in more detail in $\S 5$. 


$$
Z_{r m s}=\int_{0}^{\infty} E_{Z}\left(\kappa_{1}\right) d \kappa_{1} .
$$

In analogy with the individual dye concentrations, the measurements of $Z$ were limited to scales larger than or equal to the Kolmogorov scale. A typical measurement of $E_{Z}\left(\kappa_{1}\right)$ (measured at the centreline for $\operatorname{Re}=10600$ ) is plotted in figure 11a) as a function of $\kappa_{1} \eta$ (where $\eta$ is the Kolmogorov length scale). Because the Schmidt number of each scalar employed herein was much greater than one, it would have been necessary to resolve the Batchelor scale of the less diffusive scalar (i.e., the smaller of the two Batchelor scales) to obtain the entire spectrum of $Z$. Since this was not the case, and given that the Kolmogorov microscale was resolved at each Reynolds number, a surrogate for $Z_{r m s}$ was calculated by integrating $E_{Z}\left(\kappa_{1}\right)$ up to the Kolmogorov scale:

$$
Z_{r m s, \text { meas }}=\int_{0}^{\eta} E_{Z}\left(\kappa_{1}\right) d \kappa_{1}=\int_{0}^{1} E_{Z}\left(\kappa_{1} \eta\right) d\left(\kappa_{1} \eta\right) .
$$

$Z_{r m s, \text { meas }}$ i) provides a consistent method to compare $Z_{r m s}$ values for the various Reynolds numbers studied, and ii) quantifies the intensity of the differential diffusion effect for scales larger than the Kolmogorov scale. It is also worth nothing that, though $Z_{r m s, m e a s}$ is a surrogate to $Z_{r m s}$ (and that $Z_{r m s, m e a s}$ will always be smaller than $Z_{r m s}$ ), the contribution to $Z_{r m s}$ from concentration fluctuations smaller than the Kolmogorov scale (and larger than the Batchelor scale) should not depend on Reynolds number, but only on the Schmidt numbers of the two scalars. Therefore, assuming universal smallscale behaviour of the spectrum of $Z$, it is reasonable to assume that $Z_{r m s}-Z_{r m s, \text { meas }}$ is a constant that is only a function of the Schmidt numbers of the two scalars. That being said, the assumption of a universal small-scale shape of the spectrum of $Z$ may not be valid, as will be discussed in $\S 5.2$.

All values of $Z_{r m s, m e a s}$ presented herein are calculated according to the above defini- 
Differential diffusion of high-Schmidt-number passive scalars in a turbulent jet 25 tion. For simplicity's sake, the notation $Z_{r m s, m e a s}$ will be abbreviated by $Z_{r m s}$ from this point on.

\subsubsection{Measurements of $Z_{r m s}$}

Figure 12 plots the Reynolds number dependence of $Z_{r m s}$ at the jet centreline. For each Reynolds number, the value of $Z_{r m s}$ was determined by averaging values from multiple experiments. The error bars correspond to $95 \%$ confidence intervals (with the symbol centred on the average value of $Z_{r m s}$ ). These results indicate a decrease in the intensity of the differential diffusion as the Reynolds number is increased. Nevertheless, such effects remain non-negligible, even at the highest Reynolds number studied $(\operatorname{Re}=10600)$, for which $Z_{r m s}=0.06$.

An extrapolation of the data in figure 12 to lower Reynolds numbers is consistent with the results obtained by Saylor and Sreenivasan (1998) for scalars with similar Schmidt numbers $\left(S c_{1}=1200\right.$ and $\left.S c_{2}=4400\right)$. (Saylor and Sreenivasan (1998) found $Z_{r m s}=0.11$ for $\mathrm{Re}=430$.) Though the results are consistent, two differences in the experiments must be noted. Firstly, Saylor and Sreenivasan (1998) resolved scales down to the Batchelor scale (as compared to the Kolmogorov scale in the present study). Secondly, the measurements in the two works were obtained at different downstream distances: $x / d=50$ herein, as compared to $x / d=147$ in Saylor and Sreenivasan (1998). It is reasonable to assume that $Z_{r m s}$ decreases with downstream distance, as also observed numerically by Bilger and Dibble (1982) and Kerstein (1990), and as observed experimentally by Smith et al. (1995a) and Brownell and Su (2004, 2008). Therefore, the higher resolution of the experiments of Saylor and Sreenivasan (1998) should lead to larger values of $Z_{r m s}$, whereas their larger downstream distance should lead to smaller values of $Z_{r m s}$. These two effects may possibly offset each other, resulting in the relative agreement between the two sets of data. 
The best fit power law in figure 12 indicates that $Z_{r m s}$ scales as $R e^{-0.09}$. Given that the Reynolds number dependence of differential diffusion has never previously been measured, this result can only be compared with the predictions of different models. The present $\sim R e^{-0.1}$ dependence is closest to the $Z_{r m s} \propto R e^{-0.15}$ prediction of Fox (1999). Kerstein et al. (1995) predicted $Z_{r m s} \propto R e^{-0.25}$. This being said, the Reynolds number dependence of the true (i.e., entirely resolved) value of $Z_{r m s}$ must be even shallower than what is measured herein because the decay exponent of $Z_{r m s, \text { measured }}+Z_{r m s, \text { unresolved }}$ tends to zero as $Z_{r m s, \text { unresolved }}$ (which, as mentioned above, is likely a constant) tends to infinity. Thus, the -0.09 scaling exponent is a lower bound.

The radial dependence of differential diffusion (at each Reynolds number) was studied next. Figure 13 plots $Z_{r m s}$ as a function of $r / d$ for all Reynolds numbers. Over the range of radial positions studied in the present work $(0 \leqslant r / d \leqslant 7.5)$, differential diffusion effects were found to increase with $r / d$, regardless of the Reynolds number.

Previous studies have also observed increasing $Z_{r m s}$ with radial position. Bilger and Dibble (1982) first predicted an off-axis peak of $Z_{r m s}$ (which increasingly shifted away from the centreline with downstream position) in their modeling of a turbulent jet. When applying a linear-eddy model to simulate a turbulent jet of $H_{2}$ and propane, Kerstein (1990) observed an off-axis peak of $Z_{r m s}$ that also shifted away from the centreline with downstream position. Due to experimental constraints $\dagger$, the measurements herein did not extend to sufficiently large radial locations to observe an off-axis peak of $Z_{r m s}$. (A peak of $Z_{r m s}$ must eventually occur, as differential diffusion effects become non-existent in the ambient fluid outside the jet, where $C_{1}, C_{2}$, and therefore $Z$, are always zero.)

$\dagger$ At large $r / d$, the jet becomes so intermittent that exceptionally long data sets are required to ensure convergence of the data. Such long experiments are not possible because jet fluid (that had already passed through the jet) would be re-entrained into the jet given that the tank in which these experiments were conducted, though large, is not infinite in size. 


\subsection{PDFs of $Z$}

PDFs of $Z$ were calculated for every Reynolds number and radial position studied. Figure 14 plots PDFs of $Z$, along with their respective skewnesses $\left(S_{Z} \equiv \frac{\left\langle Z^{3}\right\rangle}{\left\langle Z^{2}\right\rangle^{3 / 2}}\right)$ and kurtoses $\left(K_{Z} \equiv \frac{\left\langle Z^{4}\right\rangle}{\left\langle Z^{2}\right\rangle^{2}}\right)$, for all five Reynolds numbers studied (Re $=900,2100,4300$, 6700, and 10600). The figure contains PDFs of $Z$ at two locations: (a) r/d $=0$, and (b) $\mathrm{r} / \mathrm{d}=6.25$. The solid curve in the figures is a standardized normal Gaussian distribution, $f(V)=\frac{1}{\sqrt{2 \pi}} \exp \left(-\frac{V^{2}}{2}\right)$, for which $S_{V}=0$ and $K_{V}=3$. In addition, the Reynolds number dependence of $S_{Z}$ and $K_{Z}$ for $r / d=0$ and 6.25 is plotted in figure 15 .

Clear differences in the structure of the PDFs emerge as either the radial position or Reynolds number changes. Regardless of Reynolds number, the off-axis $(r / d>0)$ PDFs of $Z$ exhibit larger $S_{Z}$ and $K_{Z}$ than the corresponding centreline PDFs. For instance, at Re $=10600$, the centreline PDF is effectively Gaussian $\left(S_{Z} \approx 0, K_{Z} \approx 3\right)$, while the off-axis PDF has a slight positive skewness $\left(S_{Z}=0.20\right)$ and is super-Gaussian $\left(K_{Z}=7.65>3\right)$. As the Reynolds number decreases, the centreline PDFs become increasingly positively skewed and super-Gaussian, as $S_{Z}$ increases from 0.03 for $\operatorname{Re}=10600$ to 0.16 for $\operatorname{Re}=$ 900 and $K_{Z}$ increases from 2.80 to 3.72 .

Larger values of $S_{Z}$ indicate larger probabilities of positive fluctuations of Z, which are found to occur away from the centreline and for lower Reynolds numbers. Similarly, the increased values of $K_{Z}$ indicate more frequent occurrences of large fluctuations of $Z$. These phenomena are consistent with the hypothesis, first put forth by Kerstein (1990), that differential diffusion effects increase at interfaces between parcels of jet fluid and ambient fluid. At these interfaces, the more diffusive scalar (disodium fluorescein) will diffuse more rapidly than the less diffusive scalar (sulforhodamine 101) from the parcel of dyed jet fluid into the parcel of ambient fluid, resulting in positive $Z$ fluctuations. 
This effect is most evident at interfaces between the jet and ambient fluid, where the concentration gradient, and therefore molecular diffusion, is large.

Asymmetric PDFs of Z have been obtained in previous studies. Kerstein et al. (1989) reported asymmetric PDFs of mixture fractions of hydrogen and Freon in their turbulent jet at $\operatorname{Re}=20000$. Similar to those presented herein, the asymmetry of their PDFs was more pronounced away from the centreline. PDFs of differences in mixture fractions were calculated by Kerstein (1990) when he employed a linear-eddy model to simulate a jet of hydrogen and propane. The PDFs therein were notably asymmetric away from the centreline. Smith et al. (1995a) measured histograms of differences in mixture fractions in a turbulent jet of hydrogen and carbon dioxide. At two Reynolds numbers $(\mathrm{Re}=$ 2000 and 8000), they found histograms which exhibited increased asymmetry away from the centreline. However, the asymmetry was not as pronounced at the higher Reynolds number. Finally, Brownell and Su (2004, 2008) measured PDFs of differences in mole fractions from their jet of helium and propane at $\mathrm{Re}=1000-3000$. In the laminar region near the jet, they obtained off-axis PDFs that were asymmetric. These PDFs became increasingly symmetric with downstream distance, eventually becoming symmetric in the fully turbulent region.

Joint-PDFs (JPDFs) of the scalars were also calculated to provide further insight into differential diffusion. Contour plots of the JPDFs of disodium fluorescein, $C_{1}$, and sulforhodamine $101, C_{2}$, are shown in figure 16 for $\operatorname{Re}=10600$. Figure $16(\mathrm{a})$ corresponds to $r / d=0$ while figure 16 (b) corresponds to $r / d=6.25$. Similarly, contour plots for Re $=900$ are given in figure 17.

Were no differential diffusion effects present, the JPDF of $C_{1}$ and $C_{2}$ would be described by a straight line passing through the origin with slope of 1 (which could be considered an "equal-mixing line"). This is clearly not the case for the JPDFs displayed in figure 16 
Differential diffusion of high-Schmidt-number passive scalars in a turbulent jet

and figure 17. For the centreline measurements at $\operatorname{Re}=10600$ (figure 16(a)), the peak of the JPDF occurs at the average concentration of each scalar (i.e., $\frac{c_{1}}{c_{1, r m s}}=\frac{c_{2}}{c_{2, r m s}}=0$ ). At $r / d=6.25$, the peak of the JPDF shifts to negative fluctuations of each scalar - see figure 16(b). The peaking of the JPDF at below-average concentrations (i.e., for negative values of $c_{1}$ and $c_{2}$ ) is consistent with an increasingly intermittent flow, in which the scalar concentration will be less than its mean value more often than not. The off-axis JPDF of the scalars for $\mathrm{Re}=900$ exhibits a qualitatively similar behaviour to that of the higher Reynolds number (i.e., the JPDF peak is shifted to negative scalar fluctuations). However, the centreline JPDF at this lower Reynolds number is different than its analogue at the higher Reynolds number. There are fewer large negative fluctuations in figure 17(a) (when compared to figure 16(a)), consistent with the JPDFs from the intermittent regions near the edge of the jet - figures $16(\mathrm{~b})$ and $17(\mathrm{~b})$. These intermittent regions coincide with increased differential diffusion effects (as also observed with respect to $\left.Z_{r m s}\right)$, providing further evidence supporting the amplification of differential diffusion at interfaces of between parcels of jet and ambient fluid.

\subsection{Conditional expectations}

The expectations of $Z$ conditioned upon the individual scalar concentration fluctuations $(\langle Z \mid c\rangle)$ were calculated to further elucidate the physics underlying differential diffusion. Centreline conditional expectations of $Z$ are shown in figure 18 for each Reynolds number. Figure 18(a) plots $\left\langle Z \mid c_{1}\right\rangle$, while figure 18(b) plots $\left\langle Z \mid c_{2}\right\rangle$. From this figure, it is apparent that increased differential diffusion is associated with larger positive fluctuations of the more diffusive scalar (disodium fluorescein, $c_{1}$ ). Though $Z$ appears to tend to negative values for increasingly larger fluctuations of the less diffusive scalar (sulforhodamine 101, $c_{2}$ ), this effect is much less pronounced than the preceding observation. Furthermore, though both observations are consistent with the definition of $Z\left(Z \equiv \frac{C_{1}}{\left\langle C_{1}\right\rangle}-\frac{C_{2}}{\left\langle C_{2}\right\rangle}\right)$, it is 
of particular interest to note that i) the two trends are in no way "equal in magnitude, but opposite in sign," and ii) $Z$ is effectively independent of both $c_{1}$ and $c_{2}$ for $c_{1}, c_{2}<0$.

Off-axis conditional expectations of $Z$ were also calculated. The results, for $r / d=6.25$, are plotted in figure 19. Figure 19(a) corresponds to $\left\langle Z \mid c_{1}\right\rangle$, while figure 19(b) corresponds to $\left\langle Z \mid c_{2}\right\rangle$. The off-axis behavior of $\left\langle Z \mid c_{1}\right\rangle$ (figure 19(a)) is similar to that of the corresponding conditional expectation at the centreline (figure 18(a)). (Note the difference in scales of the ordinate in both figures.) However, there is a clear difference between $\left\langle Z \mid c_{2}\right\rangle$ near the edges of the jet (figure 19(b)) and $\left\langle Z \mid c_{2}\right\rangle$ at the centreline (figure 18(b)). In the more intermittent, off-axis regions of the jet, differential diffusion effects are most prominent when there is a positive fluctuation of the more diffusive scalar and/or a negative fluctuation of the less diffusive scalar. This is consistent with the physical explanation proposed by Kerstein (1990), though different from the observations of figure $18(\mathrm{~b})$, which indicated that $\mathrm{Z}$ was effectively independent of $c_{2}$ at the jet centreline.

\section{Discussion}

This section is divided into subsections treating two main points. Firstly, we present a discussion of the relationship between the results of $\S 4$ and the notion of increased differential diffusion at interfaces between the jet fluid and the ambient fluid. This is followed by a discussion of the spectrum of the normalized concentration difference, $E_{Z}\left(\kappa_{1}\right)$. The shape of the spectrum measured herein will be discussed and compared to previous numerical simulations.

\subsection{Increases in differential diffusion in intermittent regions}

Differential diffusion effects were more pronounced as the intermittency of the flow increased. This phenomenon was seen to occur in two instances: i) as the Reynolds number 
Differential diffusion of high-Schmidt-number passive scalars in a turbulent jet 31 decreased, and ii) as $r / d$ increased. In each case, the frequency of entrained ambient fluid reaching the measurement location went up. This leads to more frequent occurrences of interfaces between the jet fluid (containing both scalars) and the ambient fluid (containing neither).

At the interfaces between parcels of jet fluid and ambient fluid, the more diffusive scalar (disodium fluorescein) will diffuse more rapidly from the parcel of jet fluid into the parcel of ambient fluid than the less diffusive scalar (sulforhodamine 101). The result will be a positive $Z$ at these interfaces. Thus, differential diffusion is increased, resulting in larger values of $Z_{r m s}$. Furthermore, since $Z$ can be expected to be positive at these interfaces, positive $S_{Z}$ and larger $K_{Z}$ can also be expected in the more intermittent regions. The phenomenon of increasing differential diffusion with increasing $r / d$ was first described by Kerstein (1990). Subsequently, Smith et al. (1995a) attributed their measured increase in differential diffusion at large radial positions to the same phenomenon.

As shown in $\S 4$, an increase in differential diffusion with increasing radial position was also observed, regardless of Reynolds number - see figure 13. Consistent with this phenomenon, $S_{Z}$ and $K_{Z}$ both increased with radial position (see figure 14), $S_{Z}$ becoming increasingly positive and $K_{Z}$ becoming increasingly super-Gaussian.

The above effect was also observed herein for measurements at the centreline as the Reynolds number decreased. The amount of entrained ambient fluid that reaches the centreline in a turbulent jet is dependent on the Reynolds number. This effect is more pronounced at Reynolds numbers below the mixing transition $\left(R e \sim 10^{4}\right)$, above which turbulent jets become fully-developed turbulent flows (Dimotakis 2000). This phenomenon was clearly visulaized by Dimotakis, Miake-Lye, and Papantoniou (1983) in photographs of a tracer along the plane of symmetry of a jet at $R e \sim 2500$ (figure 5 therein) and at $R e \sim 10^{4}$ (figure 9 therein). 
Along the centreline, $Z_{r m s}$ was largest at the lowest Reynolds numbers - see figure 12. $S_{Z}$ was also positive and increased from 0.03 for $\operatorname{Re}=10600$ to 0.16 for $\operatorname{Re}=900$. $K_{Z}$ was slightly sub-Gaussian for $\operatorname{Re}=10600$, becoming increasingly super-Gaussian at lower Reynolds numbers (and eventually reaching $K_{Z}=3.72$ for $\mathrm{Re}=900$ ). All of these conditions are consistent with an increase in differential diffusion effects at interfaces between jet fluid and ambient fluid. Hence, with respect to differential diffusion, moving radially outward in a turbulent jet and decreasing its Reynolds number have analogous effects, as both result in more occurrences of ambient fluid parcels coming into contact with jet fluid.

Finally, other preliminary experiments (not presented here) further validate the hypothesis of Kerstein (1990). A turbulent jet (containing the same two scalars) emanating into quasi-homogeneous, isotropic turbulence with zero mean flow exhibited much stronger differential diffusion effects than the present measurements in which the jet emanates into a quiescent background. The turbulent background can only serve to increase mixing between the jet and ambient fluids, thus increasing the differential diffusion.

\subsection{Spectrum of $Z$}

The spectrum of $Z$, or difference spectrum, provides information on the scales at which differential diffusion manifests itself. The details of its shape, however, remain an open question. To date, $E_{Z}(\kappa)$ has been calculated by means of a handful of numerical simulations, and only measured once. The present subsection serves to compare the spectrum measured herein, $E_{Z}\left(\kappa_{1} \eta\right)$, with the aforementioned research.

Difference spectra have been numerically simulated in flows with decaying scalar fields (i.e., Yeung and Pope 1993; Nilsen and Kosály 1997) or flows for which the scalar fields reach a statistically steady state due to the presence of turbulent production (i.e., Kerstein et al. 1995; Yeung 1998; Yeung et al. 2000; Ulitsky et al. 2002). The spectra in these 
Differential diffusion of high-Schmidt-number passive scalars in a turbulent jet 33 studies take one of two forms, either i) decreasing over all wavenumbers, or ii) increasing with wavenumber until reaching a peak at high wavenumbers.

Yeung and Pope (1993), in a stationary flow with decaying scalar fields, obtained difference spectra that were increasing with wavenumber at early times (less than 8 eddy turnover times). However, at later times, the spectra were decreasing for all wavenumbers (figure 7 therein). Nilsen and Kosály (1997) - in a decaying flow with decaying scalar fields - calculated difference spectra that were increasing with wavenumber (figure 6 therein), similar to those at early times in Yeung and Pope (1993). However, since Nilsen and Kosály's flow decayed, they were only able to conduct their simulations for up to 3.5 eddy turnover times and never observed a change in shape of $E_{Z}(\kappa)$.

In simulations with an imposed mean scalar gradient (which acts as a source of production for the scalar fields), the calculated difference spectra have always increased with wavenumber, and exhibited a peak at the high wavenumber end of the spectrum (Kerstein et al. 1995; Yeung 1998; Yeung et al. 2000; Ulitsky et al. 2002).

A study on the spectral mechanisms of differential diffusion by Yeung (1996) explains the two different forms of the difference spectra. These simulations were performed under two conditions: with decaying scalar fields, or with an imposed uniform mean scalar gradient (acting as a source of scalar variance). In the presence of an imposed mean scalar gradient, the large scales of both scalar fields are well correlated at all times given the large-scale nature of production (and its independence from the fluid's molecular properties). Therefore, forcing correlation at large scales by means of turbulent production serves to continually suppress differential diffusion at low wavenumbers. This is consistent with difference spectra that increase with wavenumber and peak at high wavenumbers. Conversely, the simulations with decaying scalar fields did not impose any large-scale 
correlation of the two scalar fields, allowing (scalar) incoherency, originating at small scales, to propagate from high to low wavenumbers.

The present difference spectra decrease for all wavenumbers and are therefore consistent with the numerical simulations of difference spectra in flows with decaying scalar fields. Even though the scalar variance budget of turbulent jets contain both production and decay terms, the latter term dominates and the result is a decaying scalar field. Furthermore, the present spectra are also consistent with the monotonically decaying spectra of Brownell and Su (2008) - also measured in a turbulent jet. However, their Schmidt numbers were all $O(1)$ given that their jet was gaseous.

The difference spectrum presented earlier, figure 11(a), was measured at the jet centreline for $\mathrm{Re}=10600$. Figure 11(b) plots the difference spectrum for a Reynolds number of 900 , measured at $r / d=6.25$. Both spectra are decreasing functions of wavenumber, consistent with what could be expected in a flow with decaying scalar fields. Spectra measured at other Reynolds numbers and radial positions (not shown) were similar.

Finally, though the difference spectra presented herein were i) decreasing functions of wavenumber, and ii) similar to those calculated in numerical simulations of decaying flows, there remain significant differences between our experiments and the previous numerical simulations. Firstly, we measured one-dimensional spectra, whereas the previous numerical simulations presented (presumably) three-dimensional spectra. Secondly, almost all numerical simulations of differential diffusion were calculated using scalars with sub-unity Schmidt numbers. To our knowledge, the largest Schmidt number employed in a simulation of differential diffusion is 4.0 (Yeung et al., 2000). In this work, the authors show that the magnitude of the reverse transfer (from small to large scales in the scalar field) "increases strongly with Schmidt number" and that "this may become important at $S c \gg 1 . "$ 
Differential diffusion of high-Schmidt-number passive scalars in a turbulent jet

\section{Conclusions}

Differential diffusion of high-Schmidt-number scalars in a turbulent jet was investigated for jet Reynolds numbers in the range $900 \leqslant R e \leqslant 10600$. The effects of differential diffusion on statistics of the scalar concentrations were studied at a single downstream location $(x / d=50)$, by systematically varying the Reynolds number and radial position. Furthermore, novel measurements of the spectrum of the differential diffusion variable, $E_{z}\left(\kappa_{1}\right)$, were presented.

Differential diffusion was observed to slowly decay with increasing Reynolds number. $Z_{r m s}$ was shown to scale as $\sim R e^{-0.1}$, similar to the prediction of Fox (1999). However, differential diffusion effects were still non-zero past the mixing transition, as $Z_{r m s}=0.06$ for $R e=10600$. Previous experimental studies, mainly in gas-phase flows, were unable to determine a scaling law for $Z_{r m s}$ as the majority were conducted at only one or two Reynolds numbers (e.g., Long et al. 1993; Bergmann et al. 1998).

The present study has also provided further evidence to support the hypothesis that differential diffusion effects become more prominent at interfaces of jet fluid and ambient fluid, as first put forth by Kerstein (1990). This supposition was first used to explain the increase in differential diffusion effects away from the jet axis. This was noted herein, along with an increase in differential diffusion with decreasing Reynolds number. This latter observation was attributed to the increase in ambient fluid reaching the jet core at lower Reynolds numbers (Dimotakis 2000). In each instance, positive $S_{Z}$ and large $K_{Z}$ provided further evidence in support of increased differential diffusion in intermittent regions of the flow.

The power spectrum of the differential diffusion variable, $E_{Z}\left(\kappa_{1} \eta\right)$, was measured and exhibited contributions at scales larger than the Kolmogorov scale for each Reynolds number and radial position. The general shape of the spectrum, which decreased with 
increasing wavenumber, was consistent with spectra calculated by means of numerical simulations in turbulent flows with decaying scalar fields. Furthermore, it was contrasted with the spectra calculated in flows in which the scalar field was statistically steady (due to production by a mean scalar gradient in a statistically steady velocity field). Numerical simulations of spectra in such flows exhibited a distinctly different form, increasing with wavenumber, until a peak was reached at high wavenumbers.

Lastly, expectations of the differential diffusion variable, $Z$, conditioned on the individual scalar concentrations were calculated. In all instances, increased differential diffusion was associated with large positive concentration fluctuations of the more diffusive scalar (disodium fluorescein, $c_{1}$ ). However, the relationship between $Z$ and the concentration fluctuations of the less diffusive scalar (sulforhodamine 101, $c_{2}$ ) was found to depend on radial position. $Z$ was effectively independent of $c_{2}$ at the jet centreline, whereas it was strongly correlated with large, negative values of $c_{2}$ for large $r / d$. 
Differential diffusion of high-Schmidt-number passive scalars in a turbulent jet 37 Acknowledgements

We thank John Bartczak and Georges Tewfik for their superior technical assistance. We also thank Adam Wahab and Wolfram Pohl for their help with this research, and Professors Paul Dimotakis, Stephen Pope and John Saylor for their constructive comments on this work. Lastly, we are grateful to the Natural Sciences and Engineering Research Council of Canada and the Fonds québécois de la recherche sur la nature et les technologies for their generous financial support. 


\section{Appendix A: Sources of error and uncertainty analysis}

The purpose of this appendix is to i) describe the potential sources of error and their (qualitative) impact on the results, and ii) quantitatively estimate the effect of these errors on our measurements of $Z$, by means of an uncertainty analysis. It should be noted that some sources of error will not affect our measurements of $Z$ to the same extent as they affect the individual instantaneous concentrations themselves. This is because i) the advection-diffusion equation is linear in concentration (when considering passive scalars, as is the case here), ii) we only consider concentrations that are normalized by their mean, iii) $Z$ is a difference of normalized concentrations, and iv) some errors affect both concentrations in the same manner (e.g., a drift in laser power) and will therefore not affect $Z$ to the same extent, given that it is a difference of normalized quantities. (In fact, such a reduction in the effects of drifts is the inherent advantage of measuring differences, and is why structure functions were introduced to the study of turbulence by the Russian school of meteorology - see Lumley and Panofsky (1964) p. 44 for more on this subject.) The analysis that follows will be made by considering each potential source of error individually, and will indicate whether such an error will affect $Z$ to the same extent as it will affect the individual concentrations themselves. Finally, this appendix will estimate an overall uncertainty for the individual concentrations, as well as for $Z$.

\section{A.1 PMT power supply stability}

The stability of the PMT power supply is $0.01 \%$. There is a power-law relationship between PMT supply voltage and output (with power-law exponent approximately equal to 7). This therefore results in an uncertainty of $0.07 \%$ in the PMT output voltage. Given that the dye concentration and PMT output voltage are linearly related, the resulting uncertainty in measured concentration is also $0.07 \%$. PMT power supply stability will 
Differential diffusion of high-Schmidt-number passive scalars in a turbulent jet

affect both PMTs in a similar manner and will therefore affect individual concentration fluctuations more than the difference of normalized concentrations.

\section{A.2 Data acquisition board resolution}

The absolute accuracy of the National Instruments PCI-MIO-16E-4 data acquisition card used in the $\pm 5 \mathrm{~V}$ range is $5.697 \mathrm{mV}$ (or $0.06 \%$ ). That being said, the mean voltage for both channels was approximately $1.5 \mathrm{~V}$, leading to an uncertainty of $0.19 \%(=5.697 \times$ $\left.10^{-3} /(2 \times 5) \times(5 / 1.5)\right)$. The resolution of the data acquisition card affects each channel independently, and therefore equally affects $Z$.

\section{A.3 Laser power stability}

The stability of the laser power supply is $0.5 \%$. Given the linear relationship between fluorescence intensity and power of the incident radiation, as well as the linear relationship between fluorescence intensity and species concentration, the resulting uncertainty in measured concentration is also $0.5 \%$. That being said, small drifts in laser power should have a negligible effect on normalized concentration fluctuations and their differences.

\section{A.4 Optical crosstalk}

As discussed in $\S 2.3$ and $\S 3.2$, an upper bound on the optical crosstalk could be inferred from a comparison of the spectra of a given dye measured with i) only that given dye in the jet, and ii) both dyes in the jet. It was found that the difference in the normalized RMS concentration of disodium fluorescein $\left(c_{1_{\text {rms }}} /\left\langle C_{1}\right\rangle\right)$ when measured with one and both dyes was $1 \%$. The difference in the normalized RMS concentration of sulforhodamine $101\left(c_{2_{r m s}} /\left\langle C_{2}\right\rangle\right)$ when measured with one and both dyes was $0.1 \%$. These differences cannot be entirely attributed to optical crosstalk given that they could only be determined from two separate experiments. Therefore some of the difference arises from other factors that affect the repeatability, but that may not be attributed to optical crosstalk. This potentially includes differences due to additional trapping caused by the presence 
of another dye in the surrounding fluid. (An estimate of trapping follows.) However, as an upper bound, the uncertainty attributable to optical crosstalk in the disodium fluorescein concentration fluctuations can be stated as $1 \%$. Similarly, the upper bound on the uncertainty attributable to optical crosstalk in the sulforhodamine 101 concentration fluctuations can be given as $0.1 \%$. Optical crosstalk affects each channel independently, and therefore affects $Z$ to a similar extent.

\section{A.5 Photobleaching and thermal blooming}

A procedure, similar to that of Saylor (1995), was developed to determine the photobleaching and thermal blooming time scales of disodium fluorescein and sulforhodamine 101 for the conditions herein. Photobleaching can have an effect on the detected signal as the fluorescence emitted by a fluorescent dye will decrease under constant laser irradiation. Thermal blooming can occur if the dyed fluid absorbs energy from the laser beam, causing an increase in its temperature. This temperature increase can lead to density differences in the fluid and also possibly cause refraction gradients which will affect the fluorescence signal (Koochesfahani 1984). Therefore, dyed fluid was irradiated with a constant laser source to determine the time for combined photobleaching and thermal blooming effects to become significant. (A test to separately measure these phenomena was not possible, though an analytical estimate of the thermal blooming is presented in $\S$ A.9.)

A glass box was designed specifically for the photobleaching / thermal blooming, trapping and attenuation tests. It was $30.5 \mathrm{~cm}$ wide by $76.2 \mathrm{~cm}$ long by $51 \mathrm{~cm}$ high, with each side consisting of a $6.35 \mathrm{~mm}$ thick panel of tempered glass. The bottom of the glass box consisted of a sheet of $2.54 \mathrm{~cm}$ thick plexiglass, while the top was open to the ambient. The box was placed inside of the large tank and served to minimize the amount of dye necessary to complete these tests. (Recall that these tests required the ambient fluid to 
Differential diffusion of high-Schmidt-number passive scalars in a turbulent jet 41 be at the same concentration as the jet and that the tank in which the experiments were performed held $9000 \ell$ of water.) When required, the jet was placed inside the box and the fluid was cycled through the jet so as to closely reproduce the experimental conditions. An outlet was located at the bottom of the box, with $1.27 \mathrm{~cm}$ diameter PVC tubing connected to it. This tubing was also connected to the $1 / 3 \mathrm{HP}$ pump which led up to the constant head reservoir. Thus, the fluid in the box could be cycled through the jet during the tests.

To perform the combined photobleaching and thermal blooming test, the glass box (which was inside the large tank) and the large tank were both filled with water. Then, one of the fluorescent dyes (disodium fluorescein or sulforhodamine 101) was mixed into the box. Once the thoroughly mixed dyed water came to rest, it was irradiated with the laser. The laser was operated at a constant power of $1.5 \mathrm{~W}$, the same power as that when conducting a turbulent jet experiment. Sampling of the fluorescence signal commenced before the laser shutter was opened, which ensured that the initial fluorescence signal was captured. The time series of the fluorescent signal was then analyzed to determine the time for its degradation.

Both disodium fluorescein and sulforhodamine 101 were tested to determine the combined photobleaching and thermal blooming time. Each test was conducted at species concentrations within their respective range (for the given measurement location) during the experiments. The fluorescent signal from disodium fluorescein was found to decrease by $10 \%$, from its peak value, in $1500 \mu \mathrm{s}$. Sulforhodamine 101 displayed minimal photobleaching and thermal blooming effects as the fluorescent signal decreased by $2 \%$ over the same time interval.

In the present work, photobleaching and thermal blooming were deemed small because the optical chopping frequency was selected such that each fluid parcel was only irradiated 
for $150 \mu \mathrm{s}$, during which the fluorescence emission of disodium fluorescein degraded by at most $1.5 \%$. Furthermore, as described in $\S 2.3$, only $100 \mu$ s of data were averaged to generate one data point. In addition, as demonstrated by Saylor (1995), chopping of the laser beam results in i) "photobleaching recovery" (i.e., reversal of photobleaching effects on the dye when not irradiated), and ii) a fourfold increase in the half-life for photobleaching (thus further minimizing its effects). It should also be noted that our measurement of the degradation of the emitted fluorescence of disodium fluorescein was performed in quiescent fluid, whereas the fluid was moving during the experiments. The latter serves to reduce photobleaching (Crimaldi, 1997; Wang and Fielder, 2000). Though we attempted to eliminate the effects of photobleaching, we are unable to accurately quantify its effects under the same conditions as our experiments (i.e., with a moving jet, chopped laser, etc.).

\section{A.6 Trapping}

Trapping occurs when the fluorescence emitted by one species is absorbed by another species. This was possible in these experiments, as the fluorescence emitted by the scalars at the measurement location could have been absorbed by dyed fluid between the measurement location and the PMTs. Therefore, tests similar to Saylor (1993) were developed to determine any trapping effects for the experimental conditions of this work.

The trapping tests were conducted in a modified version of the glass box. A $6.35 \mathrm{~mm}$ thick glass dividing wall was added to the box to create two independent compartments. The front compartment (i.e., closest to the PMTs) had dimensions of $21.6 \mathrm{~cm}$ by 30.5 cm by $51 \mathrm{~cm}$, while the back compartment had dimensions of $54.5 \mathrm{~cm}$ by $30.5 \mathrm{~cm}$ by $51 \mathrm{~cm}$. This modified glass box was placed inside the large tank to reduce the required amount of fluorescent dye and closely resemble experimental conditions.

To begin each trapping test, given concentrations of the dyes were mixed into the back 
Differential diffusion of high-Schmidt-number passive scalars in a turbulent jet 43 compartment of the modified box. The jet was also placed in the back compartment with dyed fluid constantly cycling through it, so as to reproduce the experimental conditions as closely as possible, and to also minimize any effects of photobleaching and thermal blooming - see $\S$ A.5. The optical chopper was also on during these tests to further minimize any photobleaching or thermal blooming. The dyed fluid was then irradiated with the laser, at a power of $1.5 \mathrm{~W}$, and the two subsequent PMT voltages were measured and retained as reference values. Next, the same concentrations of fluorescent dyes were mixed into the water in the front compartment (located between the PMTs and the back compartment). Again, the back compartment was irradiated with the laser and the fluorescence was measured. This second measurements of the PMT voltages were then compared to the reference values (from the tests with no dye in the front compartment). Any differences in the two signals could therefore be attributed to the additional trapping from the dyes added to the front compartment. This difference will, however, overestimate any trapping effects, since the mean axial concentration decays in the downstream direction, whereas the dye concentration in both sections of the glass box was constant.

The difference in the trapped and "untrapped" PMT voltages was at most $0.03 \mathrm{~V}$, resulting in an uncertainty of $2 \%$ (because the average PMT voltages in these tests were approximately $1.5 \mathrm{~V}$ ). However, trapping should have a negligible effect on normalized concentration differences because the fluorescence of both dyes passes through the same concentration fields on its way from the measurement volume to the PMTs.

\section{A.7 Laser attenuation}

As the laser beam passes through dyed fluid, it will attenuate as its energy is absorbed by the fluorescent tracer. If the laser beam passes through different amounts of dyed fluid before reaching the measurement location throughout the course of an experiment (as in the case of a turbulent jet) then the laser intensity may vary at this location. Therefore, 
a test was developed to determine if attenuation was significant for the conditions in this work.

The attenuation test was conducted with a known concentration of the dyes in the glass box. Again, the dye was cycled through the jet and the optical chopper was on, so as to prevent any photobleaching or thermal blooming effects. The average fluorescence signal was first detected with the measurement location at the centre of the glass box. Next, the test was repeated with the box shifted by $10 \mathrm{~cm}$ (keeping the jet and measurement locations stationary), such that the laser beam passed through less dyed fluid before reaching the measurement volume. The average PMT outputs from the two experiments were then compared, with any differences being attributed to attenuation. Similarly to the trapping tests, this test overestimates the effect of laser attenuation given that these tests maintain a uniform dye concentration (equal to the jet centreline value) at all locations in the glass box, whereas in the experiments, the average dye concentration decreases with increased radial distance from the jet centreline.

Attenuation tests were performed with a mixture of the two dyes at approximately the same concentrations as their respective average concentrations at the measurement location during the turbulent jet experiments. The difference in the attenuated and unattenuated PMT voltages was at most $0.02 \mathrm{~V}$, resulting in an uncertainty of $1.3 \%$ (because the average PMT voltages were approximately $1.5 \mathrm{~V}$ ). The amount of attenuation during an actual experiment can be, to a first approximation, estimated by prorating the drop in PMT output during this test by the ratio of a) the amount of dye through which the laser passes in an experiment to b) the amount of dye through which the laser passed in our test. The former was calculated assuming an analytical form of the radial concentration profile using a (mean) scalar field half-width (normalized by $x$ ) of 0.125 , which is larger than the corresponding value of 0.1 for the velocity field (Fischer et al., 1979). 
Differential diffusion of high-Schmidt-number passive scalars in a turbulent jet 45 The value of the ratio depends on the exact form of the radial concentration profile, but is always around 0.5. Therefore, the attenuation is overestimated by a factor of 2 and the uncertainty in the concentration due to laser attenuation is $0.7 \%$.

Like trapping, laser attenuation should have a negligible effect on normalized concentration differences because the laser beam of both dyes passes through very similar concentration fields on its way from the laser to the measurement volume.

\section{A.8 Buoyancy}

Buoyancy effects are possible if the jet fluid has a different density than the ambient fluid into which it issues, or if the jet fluid is heated at the measurement location, thereby resulting in a density difference. Density differences of the issuing fluid could have occurred if the jet fluid was at a different temperature than the background fluid or if the presence of the fluorescent dye caused an increase in the density of the jet fluid. Heating of the jet fluid at the measurement location was also possible from laser irradiation, i.e., thermal blooming. These effects will be discussed and analytically estimated in this and the next section.

The fluorescent dyes were added to the jet fluid in dilute concentrations. (The jet nozzle concentration for all experiments was $2 \times 10^{-7} \mathrm{~mol} / \ell$ for disodium fluorescein and $6 \times 10^{-7} \mathrm{~mol} / \ell$ for sulforhodamine 101.) The molecular weights of disodium fluorescein and sulforhodamine 101 (as given by the manufacturers) are $376.28 \mathrm{~g} / \mathrm{mole}$ and 606.71 $\mathrm{g} /$ mole, respectively. Therefore, when mixed in water $\left(\rho_{w} \approx 1000 \mathrm{~kg} / \mathrm{m}^{3}\right)$, the difference of the densities of the dyed jet fluid, at the jet nozzle (where the scalar concentrations were the greatest), and the background fluid was calculated to be $4.7 \times 10^{-5} \%$, a negligible difference.

Small buoyancy effects can eventually influence the jet at a location downstream of the 
nozzle. This distance was calculated analogously to the method of Miller and Dimotakis (1991). Using a a non-dimensional buoyancy lengthscale, $L_{b}$ (Dowling, 1988):

$$
\frac{L_{b}}{d} \equiv{\frac{\pi \rho_{j}}{4 \rho_{\infty}}}^{1 / 4}{\frac{\rho_{j} u_{o}^{2}}{\left(\rho_{j}-\rho_{\infty}\right) g d}}^{1 / 2},
$$

where $\rho_{j}$ is the jet fluid density, $\rho_{\infty}$ is the background fluid density, and $g$ is gravitational acceleration. Papanicolau and List $(1987,1988)$ found jets to be momentum dominated if $x / L_{b}$ was less than one while Chen and Rodi (1980) defined jets to be momentum dominated for a value of $x / L_{b}$ less than 0.5 . The jet fluid density used for these calculations was for a combination of disodium fluorescein, at a concentration of $2 \times 10^{-7} \mathrm{~mol} / \ell$, and sulforhodamine 101, at a concentration of $6 \times 10^{-7} \mathrm{~mol} / \ell$. These concentrations yielded a difference in the jet fluid density and background fluid density of $4.7 \times 10^{-4} \mathrm{~kg} / \mathrm{m}^{3}$. Thus, the smallest non-dimensional buoyancy lengthscale in this work was calculated to be 2643 , which occurred at a Reynolds number of 4300 when the jet diameter was $8 \mathrm{~mm}$. Therefore, the jet in this work was momentum driven for at least 1321 jet diameters downstream, using the more stringent condition of Chen and Rodi (1980). Clearly, the jet did not experience any buoyancy effects in these experiments, since measurements were taken 50 jet nozzle diameters downstream of the exit.

A density difference between the dyed fluid and background fluid could also have occurred if the two fluids were at different temperatures. This would have been possible if the dyed jet fluid was entering the background at a different temperature, or if the laser heated the dyed fluid in the measurement volume. The first possibility was eliminated by taking the water for the jet fluid from the large tank of background fluid, just prior to conducting an experiment. A mercury thermometer was also used to monitor the temperature of both fluids, ensuring that they were equal.

Lastly, any buoyancy effect would affect both dyes similarly. 


\section{A.9 Heating of the fluid by the laser}

Heating of the dyed fluid in the measurement volume by the laser beam was calculated by the same method as Miller and Dimotakis (1991). It was assumed that all of the attenuation of the laser beam, as it passed through the cross-section of the jet, went into heating the dyed fluid. For the purpose of this calculation, the maximum attenuation across the radius of the jet of largest cross-section $(9 \mathrm{~cm})$ was estimated as $1.5 \%$. Therefore, the attenuation across the measurement volume, a $100 \mu \mathrm{m}$ length, was estimated to be $0.002 \%$, assuming that the attenuation across the jet cross-section was linear. Maximum heating occurred when a parcel of fluid resided in the measurement volume for the longest time period. This coincided with the $8 \mathrm{~mm}$ diameter jet at a Reynolds number of 4300 . In this instance, the mean centreline axial velocity at the measurement location was estimated to be $0.065 \mathrm{~m} / \mathrm{s}$ (from equation (3.3)). Therefore, the residence time of a parcel of dyed fluid was approximately 0.0015 s. Approximating the measurement volume as a $100 \mu \mathrm{m}$ long cylinder with a diameter of $100 \mu \mathrm{m}$, the temperature rise of the fluid parcel was found to be $0.01^{\circ} \mathrm{C}$. Hence, heating of dyed fluid in the measurement volume was negligible and did not influence the results.

\section{A.10 PMT drift}

Instability of the output signal, or drift, can occur when using PMTs. However, PMT drift can be minimized by warming up the PMTs in the presence of a light source (as recommended by the manufacturer). This solution was implemented and the PMTs were warmed up with a light source whose intensity resulted in output voltages similar to those obtained during an experiment. A light emitting diode (LED) was used as the light source during the warm-up period (approximately 1 hour). The drift in the PMT output was estimated by measuring the change in PMT output voltage over the time of an 
experiment and was found to be $0.73 \%$. PMT drift can affect each PMT independently, and therefore affect $Z$ to a similar extent.

\section{A.11 Uncertainty estimates}

Assuming all uncertainties are independent and random, uncertainties should be added in quadrature (Taylor, 1997; Tavoularis, 2005). In other words the total uncertainty should be equal to the square-root of the sum of the squares of the individual uncertainties. Furthermore, the uncertainty of the difference of two numbers should also be the quadratic sum of the individual uncertainties of the two numbers.

Summing in quadrature all the above uncertainties in the absolute concentration leads to an uncertainty of $2.5 \%$ in the disodium fluorescein concentration, and an uncertainty of $2.3 \%$ in the sulforhodamine 101 concentration. This translates to an uncertainty in $Z$ of $3.4 \%$. However, as mentioned above, doing so (incorrectly) assumes all of the uncertainties in the measurement of $Z$ to be random and independent. Some are not. These include drift in the PMT and laser power supplies, trapping, attenuation, and buoyancy effects. The effect of these potential sources of error on $Z$ will be much smaller than their effect on the absolute concentrations due to i) the act of normalizing, and ii) the inherent benefits of performing a subtraction, as discussed at the beginning of this appendix. Therefore, the above calculation overestimates the total error. This is confirmed, for example, by the repeatability in the measurements of $Z_{r m s}$ (see figure 12), which would have had much wider $95 \%$ confidence intervals if the above uncertainty were accurate and not overestimated. A more refined estimate of the uncertainty in $Z$ (omitting the above-described non-independent uncertainties that have a negligible effect on $Z$ ) was $1.5 \%$.

Finally, it is worth mentioning that the ideal method for quantifying the uncertainty of our experimental method would have been to perform our experiment using two dyes, of 
Differential diffusion of high-Schmidt-number passive scalars in a turbulent jet 49 identical Schmidt number, but with different emission spectra. Such scalars, given their identical Schmidt number, would never differ in their concentration and $Z$ would always be zero. A measurement with such dyes would have permitted an exact estimate of our overall experimental error. We, however, were not able to find another dye with i) the same Schmidt number of either disodium fluorescein or sulforhodamine 101, and ii) a different emission spectrum. 


\section{References}

ANTONIA, R.A. \& ZHAO, Q., 2001. Effect of initial conditions on a circular jet. Exp. Fluids 31, 319-323.

BATCHELOR, G.K., 1959. Small-scale variation of convected quantities like temperature in turbulent fluid. Part 1. General discussion and the case of small conductivity. $J$. Fluid Mech. 5, 113-133.

BENSON, D.M., BRYAN, J., PLANT, A.L., GOTTO, A.M., \& SMITH, L.C., 1985. Digital imaging fluorescence microscopy: Spatial heterogeneity of photobleaching rage constants in individual cells. J. Cell Biol. 100, 1309-1323.

BERGMANN, V., MEIER, W., WOLFF, D., \& STRICKER, W., 1998. Application of spontaneous Raman and Rayleigh scattering and 2D LIF for the characterization of a turbulent $\mathrm{CH}_{4} / \mathrm{H}_{2} / \mathrm{N}_{2}$ jet diffusion flame. Appl. Phys. B 66, 489-502.

BILGER, R.W., 1977. Reaction rates in diffusion flames. Comb. and Flame 30, 277284 .

BILGER, R.W., 1981. Molecular transport effects in turbulent diffusion flames at moderate Reynolds number. AIAA J. 20, 962-970.

BILGER, R.W. \& DIBBLE, R.W., 1982. Differential molecular diffusion effects in turbulent mixing. Combust. Sci. Tech. 28, 161-172.

BROWNELL, C.J. \& SU, L.K., 2004. Planar measurements of differential diffusion in turbulent jets. AIAA Paper 2004-2335 34 ${ }^{\text {th }}$ AIAA Fluid Dynamics Conference.

BROWNELL, C.J. \& SU, L.K., 2008. Planar laser imaging of differential molecular diffusion in gas-phase turbulent jets. Phys. Fluids 20, Art. 035109, 1-20.

CHEN, C.J. \& RODI, W., 1980. Vertical turbulent buoyant jets: A review of the experimental data. Pergamon Press. 
Differential diffusion of high-Schmidt-number passive scalars in a turbulent jet 51 CRIMALDI, J.P., 1997. The effect of photobleaching and velocity fluctuations on single-point LIF measurements. Exp. Fluids 31, 90-102.

DIBBLE, R.W. \& LONG, M.B., 2005. Investigation of differential diffusion in turbulent jet flows using planar laser Rayleigh scattering. Combust. and Flame 143, 644-649.

DIMOTAKIS, P.E., MIAKE-LYE, R.C., \& PAPANTONIOU, D.A., 1983. Structure and dynamics of round turbulent jets. Phys. Fluids 26, 3185-3192.

DIMOTAKIS, P.E., 2000. The mixing transition in turbulent flows. J. Fluid Mech. 409, 69-98.

DIMOTAKIS, P.E., 2005. Turbulent mixing. Annu. Rev. Fluid Mech. 37, 329-356.

DOWLING, D.R., 1988. Mixing in gas phase turbulent jets. PhD dissertation, California Institute of Technology.

DOWLING, D.R. \& DIMOTAKIS, P.E., 1990. Similarity of the concentration field of gas-phase turbulent jets. J. Fluid Mech. 218, 109-141.

DOWLING, D.R., LANG, D.B. \& DIMOTAKIS, P.E., 1989. An improved laser-Rayleigh scattering photodetection system. Exp. Fluids 7, 435-440.

DRAKE, M.C., PITZ, R.W., \& LAPP, M., 1986. Laser measurements on nonpremixed $\mathrm{H}_{2}$-air flames for assessment of turbulent combustion models. AIAA Journal 24, 905-917. FERDMANN, E., ÖTÜGEN, M.V., \& KIM, S., 2000. Effect of the initial velocity profile on the development of round jets. J. Propulsion Power 16, 676-686.

FISCHER, H.B., LIST, E.J, KOH, R.C.Y., IMBERGER, J \& BROOKS, N.H., 1979. Mixing in inland and coastal waters, Academic Press.

FOX, R.O., 1999. The Lagrangian spectral relaxation model for differential diffusion in homogeneous turbulence. Phys. Fluids 11, 1550-1571.

FRIEHE, C.A., VAN ATTA, C.W., \& GIBSON, C.H., 1971. Jet turbulence: dissipation 
Hamamatsu PhOTONICS, 2006. Photomultiplier Tubes, Online Catalog.

JENKINS, F.A. \& WHITE, H.E., 1957. Fundamentals of Optics, McGraw Hill.

KERSTEIN, A.R., DIBBLE, R.W., LONG, M.B., YIP, B., \& LYONS, K., 1989. Measurement and computation of differential molecular diffusion in a turbulent jet. In Proc. $7^{\text {th }}$ Symp. on Turb. Shear Flows, paper 14-2.

KERSTEIN, A.R., 1990. Linear-eddy modelling of turbulent transport. Part 3. Mixing and differential molecular diffusion in round jet. J. Fluid Mech. 216, 411-435.

KERSTEIN, A.R., CREMER, M.A., \& MCMURTRY, P.A., 1995. Scaling properties of differential molecular diffusion effects in turbulence. Phys. Fluids 7, 1999-2007.

KOLMORGOROV, A.N., 1941. Dokl. Akad. Nauk SSSR 30, 301.

KOOCHESFAHANI, M., 1984. Experiments on turbulent mixing and chemical reactions in a liquid mixing layer. $\mathrm{PhD}$ dissertation, California Institute of Technology.

KRONENBURG, A. \& BILGER, R.W., 1997. Modelling of differential diffusion effects in nonpremixed nonreacting turbulent flow. Phys. Fluids 9, 1435-1447.

KRONENBURG, A. \& BILGER, R.W., 2001a. Modelling differential diffusion in nonpremixed reacting turbulent flow: application to turbulent jet flames. Combust. Sci. Tech. 166, $175-194$.

KRONENBURG, A. \& BILGER, R.W., 2001b. Modelling differential diffusion in nonpremixed reacting turbulent flow: model development. Comb. Sci. Tech. 166, 198-227.

LAVERTU, T.M., 2006. Differential diffusion in a turbulent jet. PhD dissertation, McGill University.

LONG, M.B., STARNER, S.H., \& BILGER, R.W., 1993. Differential diffusion in jets using joint PLIF and Lorenz-Mie imaging. Comb. Sci. Tech. 92, 209-224. 
Differential diffusion of high-Schmidt-number passive scalars in a turbulent jet 53 LUMLEY, J.L. \& PANOFSKY, H.A., 1964. The structure of atmospheric turbulence Wiley.

MASRI, A.R., DIBBLE, R.W., \& BARLOW, R.S., 1992. Chemical kinetic effects in nonpremixed flames of $\mathrm{H}_{2} / \mathrm{CO}_{2}$ fuel. Comb. and Flame 91, 285-309.

MEIER, W., PRUCKER, S., CAO, M.H., \& STRICKER, W., 1996. Characterization of turbulent $\mathrm{H}_{2} / \mathrm{N}_{2} /$ Air jet diffusion flames by single-pulse spontaneous Raman scattering. Comb. Sci. Tech. 118, 293-312.

MILLER, P.L., 1991. Mixing in high Schmidt number turbulent jets. PhD dissertation, California Institute of Technology.

MILLER, P.L. \& DIMOTAKIS, P.E., 1991a. Stochastic geometric properties of scalar interfaces in turbulent jets, 1991. Phys. Fluids A - Fluid Dynamics 3, 168-177.

MILLER, P.L, \& DIMOTAKIS, P.E., 1991b. Reynolds number dependence of scalar fluctuations in a high Schmidt number turbulent jet. Phys. Fluids A - Fluid Dynamics 3, 1156-1163.

MILLER, P.L. \& DIMOTAKIS, P.E., 1996. Measurements of scalar power spectra in high Schmidt number turbulent jets. J. Fluid Mech. 308, 129-146.

NILSEN, V. \& KOSÁLY, G., 1997. Differentially diffusing scalars in turbulence. Phys. Fluids 9, 3386-3397.

PANCHAPAKESAN, N.R. \& LUMLEY, J.L., 1993. Turbulence measurements in axisymmetric jets of air and helium. Part 2. Helium jet. J. Fluid Mech. 246, 225-247.

PAPANICOLAOU, P.N. \& LIST, E.J., 1987. Statistical and spectral properties of tracer concentration in round buoyant jets. J. Heat Mass Transfer 30, 2059-2071.

PAPANICOLAOU, P.N. \& LIST, E.J., 1988. Investigations of round vertical turbulent buoyant jets. J. Fluid Mech. 195, 341-391. 
PITSCH, H. \& PETERS, N., 1998. A consistent flamelet formulation for non-premixed combustion considering differential diffusion effects. Comb. and Flame 114, 26-40.

PITSCH, H., 2000. Unsteady flamelet modelling of differential diffusion in turbulent jet diffusion flames. Comb. and Flame 123, 358-374.

PRESS, W.H., TEUKOLSKY, S.A., VETTERLING, W.T., \& FLANNERY, B.P., 1992. Numerical recipes in FORTRAN 77, second edition Cambridge University Press.

READY, J.F., 1978. Industrial Applications of Lasers Academic Press.

ROSSI, B., 1957. Optics, Addison-Wesley.

SAHAR, E. \& TREVES, D., 1977. Bleaching and diffusion of laser dyes in solution under high power UV irradiation. Optics Communications 21, 20-24.

SAYLOR, J.R., 1993. Differential diffusion in turbulent and oscillatory, non-turbulent, water flows. PhD dissertation, Yale University.

SAYLOR, J.R., 1995. Photobleaching of disodium fluorescein in water. Exp. Fluids 18, $445-447$.

SAYLOR, J.R. \& SREENIVASAN, K.R., 1998. Differential diffusion in low Reynolds number water jets. Phys. Fluids 10, 1135-1146.

SHRAIMAN, B.I. \& SIGGIA, E.D., 2000. Scalar turbulence. Nature 405, 639-646.

SMITH, L.L., DIBBLE, R.W., TAlBOT, L., BARLOW, R.S., \& CARTER, C.D., 1995a. Laser Raman scattering measurements of differential molecular diffusion in nonreacting turbulent jets of $\mathrm{H}_{2} / \mathrm{CO}_{2}$ mixing with air. Phys. Fluids 7, 1455-1466.

SMITH, L.L., DiBBle, R.W., TAlbot, L., BARlOW, R.S., \& CARTER, C.D., 1995b. Laser Raman scattering measurements of differential molecular diffusion in turbulent nonpremixed jet flames of $\mathrm{H}_{2} / \mathrm{CO}_{2}$ fuel. Comb. and Flame 100, 153-160.

SREENIVASAN, K.R., 1991. On local isotropy of passive scalars in turbulent shear flows. Proc. R. Soc. Lond. A 434, 165-182. 
Differential diffusion of high-Schmidt-number passive scalars in a turbulent jet 55 TAVOULARIS, S., 2005. Measurement in fluid mechanics. Cambridge University Press. TAYLOR, J.R., 1997. An introduction to error analysis: The study of uncertainties in physical measurements, Second edition. University Sciencebooks.

TSUJI, H. \& YAMAOKA, I., 1971. Structure analysis of counterflow diffusion flames in the forward stagnation region of a porous cylinder. Thirteenth Symp. (Intl.) on Combustion, 723 .

ULITSKY, M., VAITHIANATHAN, T., \& COLLINS, L.R., 2002. A spectral study of differential diffusion of passive scalars in isotropic turbulence. J. Fluid Mech. 460, 1-38.

WANG, G.R. \& FIEDLER, H.E., 2000. On high spatial resolution scalar measurement with LIF. Part 1: Photobleaching and thermal blooming. Exp. Fluids 29, 257-264.

WARE, B.R., CYR, D., GORTI, S., \& LANNI, F., 1983. Electrophoretic and frictional properties of particles in complex media measured by laser light scattering and fluorescence photobleaching recovery. In Measurement of Suspended Particles by Quasi-Elastic Light Scattering, 255-289.

WARHAFT, Z., 2000. Passive scalars in turbulent flows. Annu. Rev. Fluid Mech. 32, 203-240.

WIENER, N., 1949. Extrapolation, interpolation, and smoothing of stationary time series Technology Press.

YEUNG, P.K. \& POPE, S.B., 1993. Differential diffusion of passive scalars in isotropic turbulence. Phys. Fluids A 5, 2467-2478.

YEUNG, P.K., 1996. Multi-scalar triadic interactions in differential diffusion with and without mean scalar gradients. J. Fluid Mech. 321, 235-278.

YEUNG, P.K., 1998. Correlations and conditional statistics in differential diffusion: Scalars with uniform mean scalar gradients. Phys. Fluids 10, 2621-2635. 
YEUNG, P.K., SYKES, M.C., \& VEDULA, P., 2000. Direct numerical simulation of differential diffusion with Schmidt numbers up to 4.0. Phys. Fluids 12, 1601-1604.

XU, G. \& ANTONIA, R.A., 2002a. Effect of different initial conditions on a turbulent round free jet. Exp. Fluids 33, 677683.

XU, G. \& ANTONIA, R.A., 2002b. Effect of initial conditions on the temperature field of a turbulent round free jet. Int. Comm. Heat Mass Transfer 29, 1057-1068. 
$\operatorname{Re} \mathrm{d}(\mathrm{mm}) \quad \eta(\mu \mathrm{m}) \eta_{b, 1}(\mu \mathrm{m}) \eta_{b, 2}(\mu \mathrm{m})$

\begin{tabular}{ccccc}
\hline 10600 & 8 & 145 & 3.3 & 2.1 \\
6700 & 8 & 205 & 4.6 & 2.9 \\
4300 & 8 & 286 & 6.4 & 4.1 \\
4300 & 3.81 & 136 & 3.0 & 1.9 \\
2100 & 3.81 & 233 & 5.2 & 3.3 \\
2100 & 1.6 & 98.0 & 2.2 & 1.4 \\
900 & 1.6 & 185 & 4.1 & 2.6 \\
\hline
\end{tabular}

TABLE 1. Centreline Kolmogorov $(\eta)$ and Batchelor $\left(\eta_{b}\right)$ length scales for each combination of Reynolds number and jet diameter. $\eta_{b, 1}$ corresponds to the Batchelor scale of disodium fluorescein $\left(S c_{1}=2000\right)$, and $\eta_{b, 2}$ corresponds to that of sulforhodamine $101\left(S c_{2}=5000\right)$. 


\begin{tabular}{cccc}
\hline $\operatorname{Re}$ & $\mathrm{d}(\mathrm{mm})$ & $f_{s}$ & $f_{\eta}(\mathrm{Hz})$ \\
\hline 10600 & 8 & 1606 & 176 \\
6700 & 8 & 1015 & 79 \\
4300 & 8 & 651 & 36 \\
4300 & 3.81 & 1368 & 160 \\
2100 & 3.81 & 668 & 46 \\
2100 & 1.6 & 1591 & 258 \\
900 & 1.6 & 682 & 59 \\
\hline
\end{tabular}

TABLE 2. Centreline sampling $\left(f_{s}\right)$ and Kolmogorov $\left(f_{\eta}\right)$ frequencies for each combination of Reynolds number and jet diameter. 


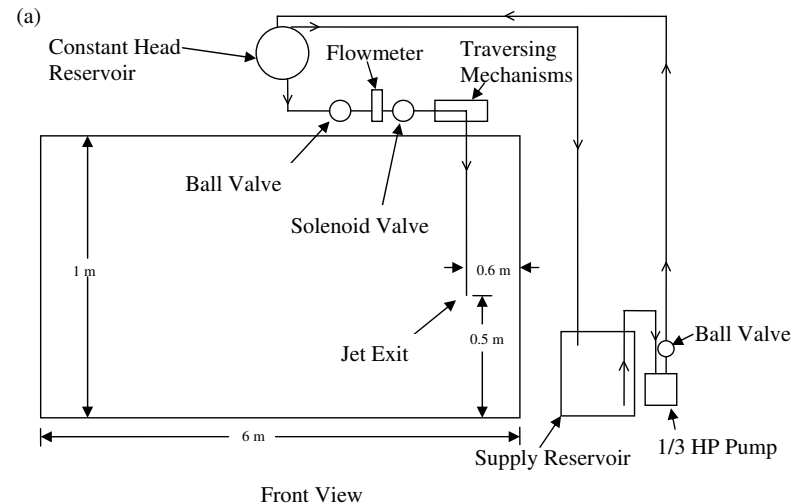

(b)

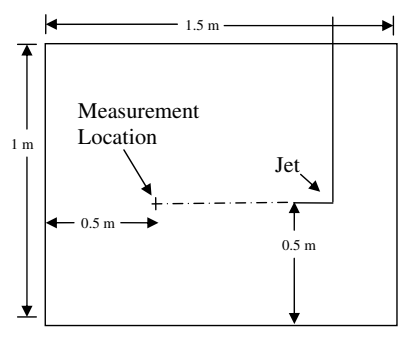

Side View

FiguRE 1. Schematic of the supply reservoir, constant head reservoir and jet components. a) Front view. b) Side view. 
(a)
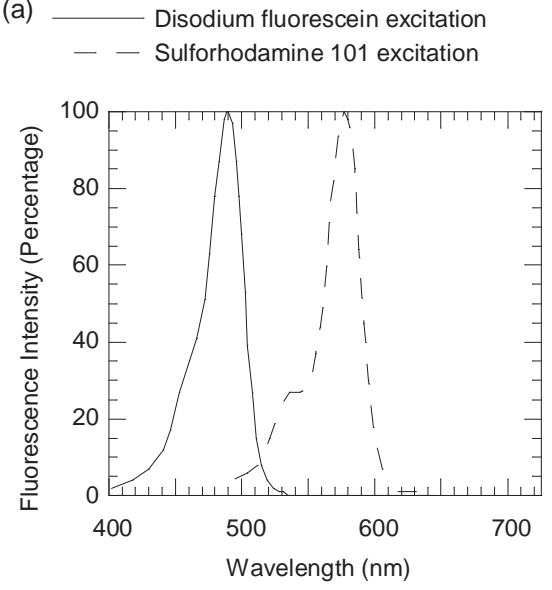

(b)
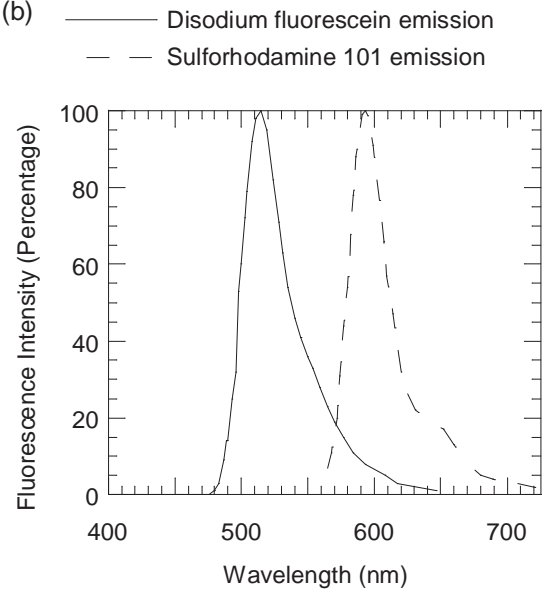

FIGURE 2. Disodium fluorescein and sulforhodamine 101 fluorescence spectra: (a) excitation spectra, and (b) emission spectra (given an excitation wavelength of $488 \mathrm{~nm}$ ). The disodium fluorescein data (solid lines) are from the measurements of Saylor and Sreenivasan (1998). The sulforhodamine 101 data (dashed lines) are provided by the manufacturer. 


\section{Page 61 of 76}

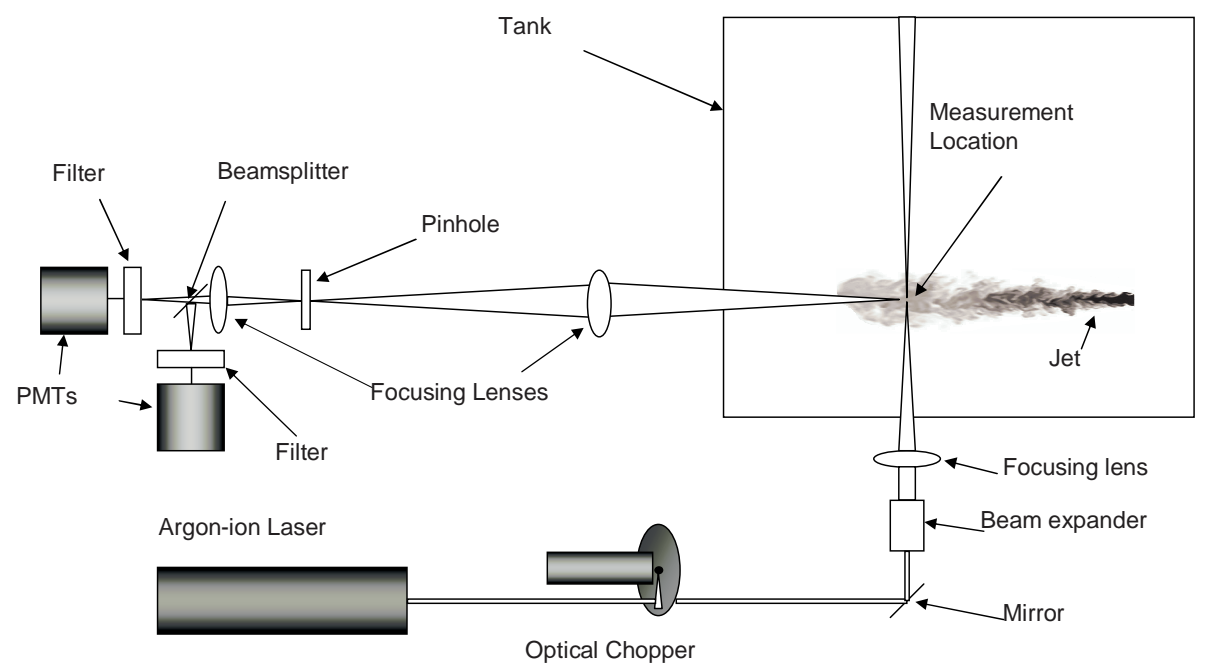

FIgURE 3. Schematic diagram of the laser focusing and signal detection optics - top view. 


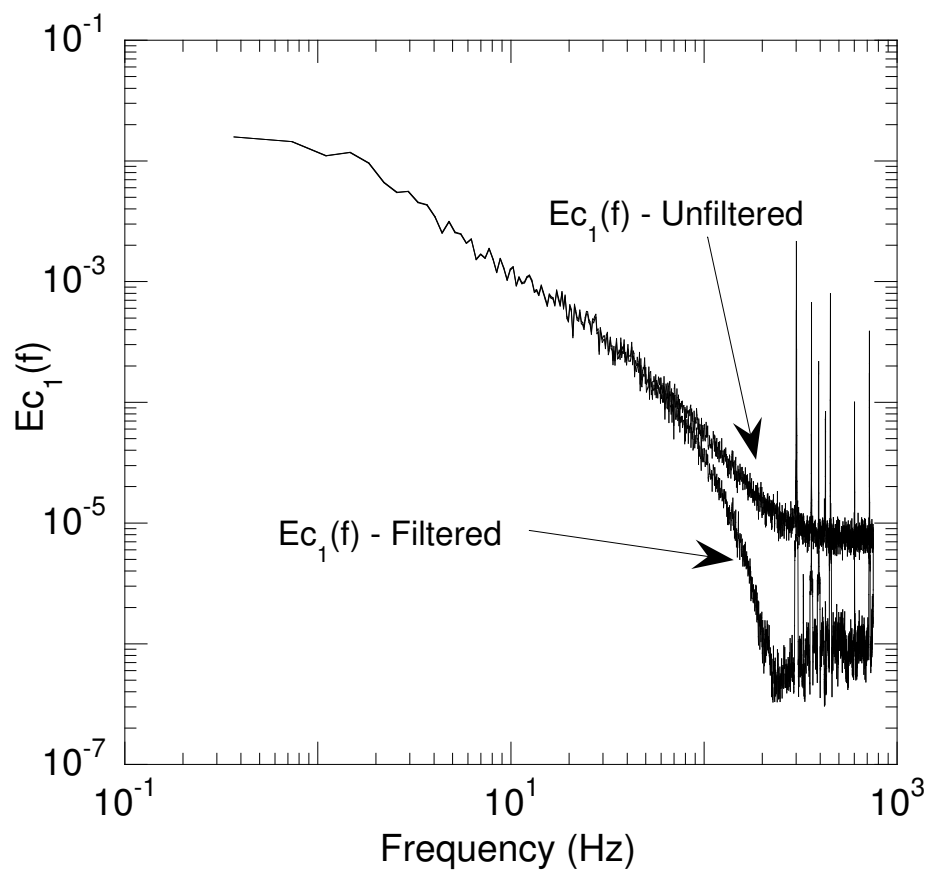

Figure 4. Comparison of disodium fluorescein spectra, $\mathrm{Ec}_{1}(\mathrm{f})$, at $\mathrm{Re}=10600$ before and after the application of the Wiener filter. 


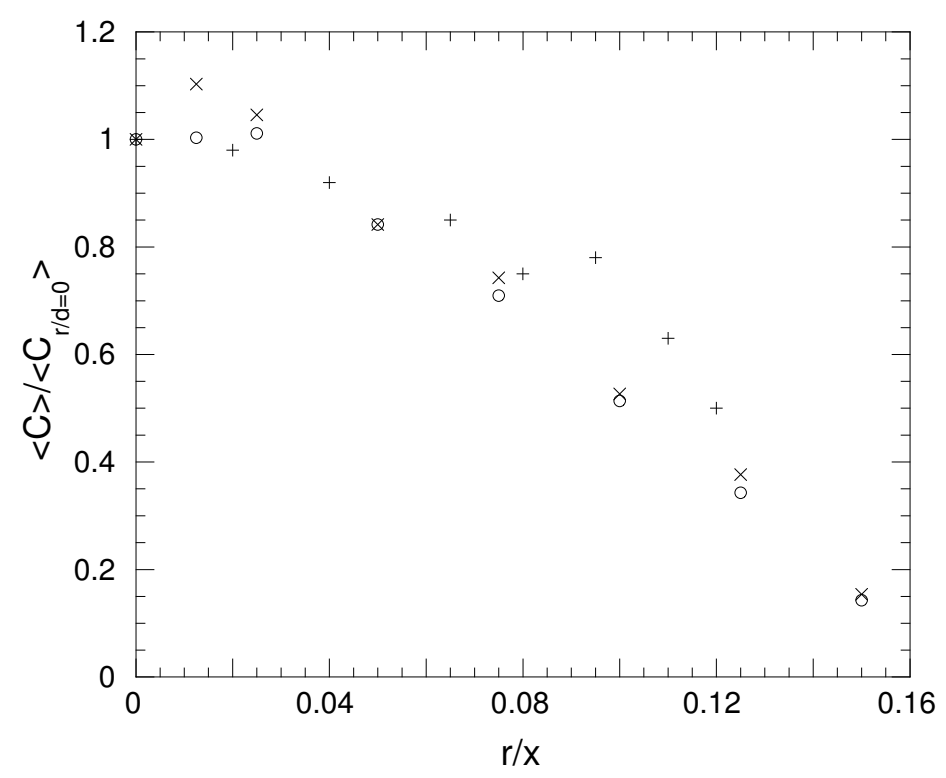

FIGURE 5. Radial variation of individual normalized average scalar concentrations of disodium fluorescein (o) and sulforhodamine $101(\times)$ from the present study (Re =10600), and of helium (+) from Panchapakesan and Lumley (1993). 


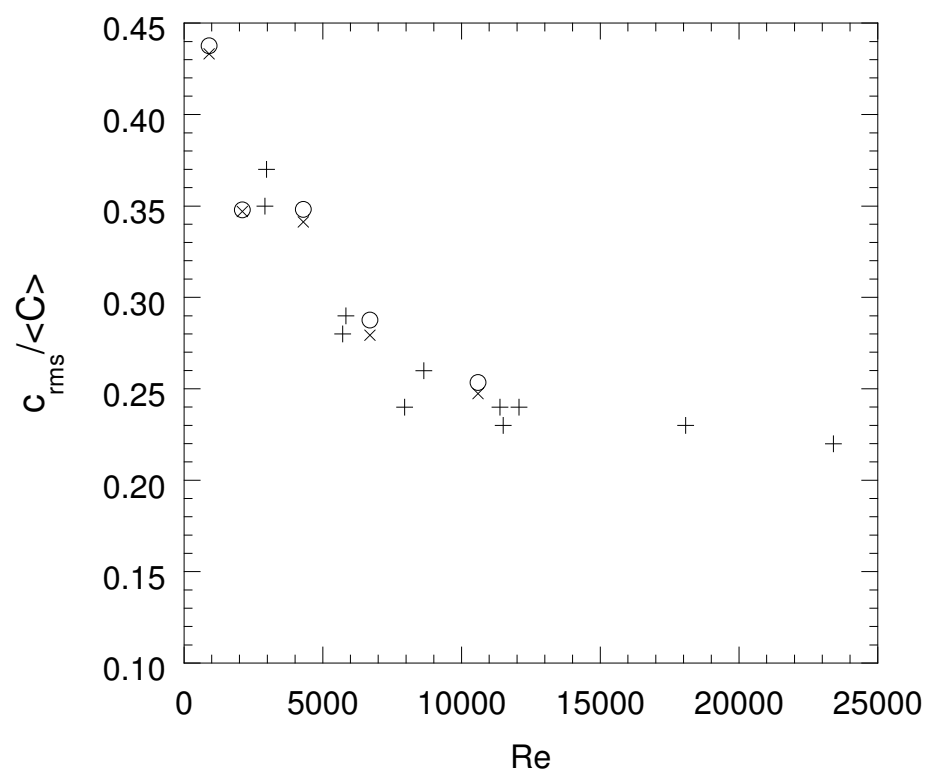

FiguRE 6. Reynolds number dependence of the normalized RMS scalar fluctuation of disodium fluorescein $\left(S c_{1}=2000, \circ\right)$ and sulforhodamine $101\left(S c_{2}=5000, \times\right)$ along the jet centreline. The + symbols are the high-Schmidt-number $(S c=1900)$ turbulent jet data of Miller (1991) and Miller and Dimotakis (1991). 


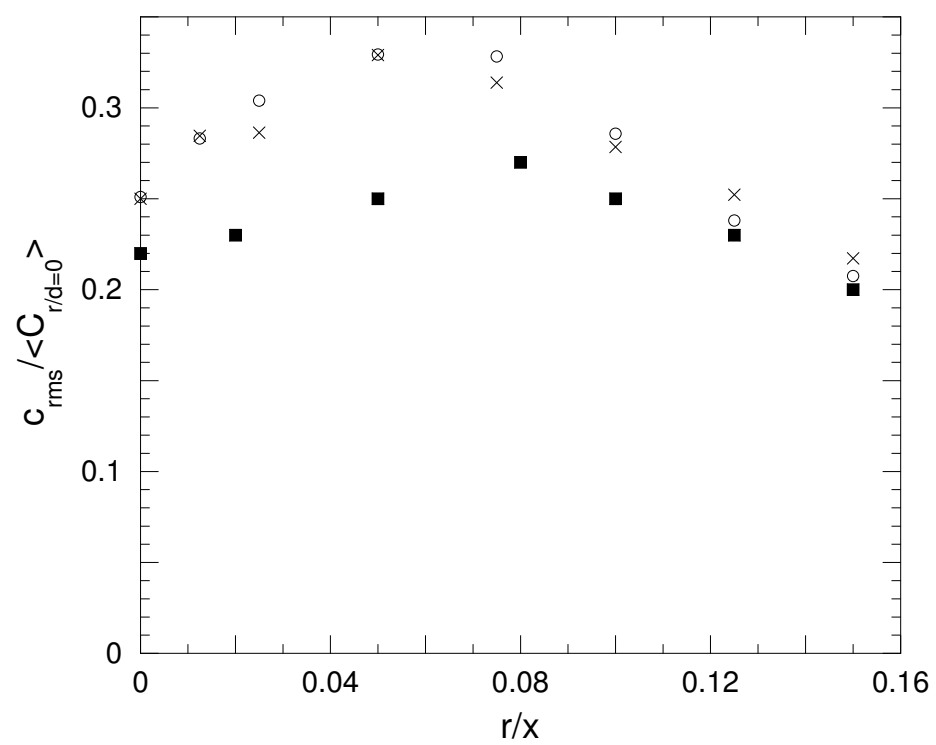

FiguRE 7. Radial profile of normalized RMS scalar concentration fluctuations of disodium fluorescein $\left(S c_{1}=2000, \circ\right)$ and sulforhodamine $101\left(S c_{2}=5000, \times\right)$ from the present study (Re $=10600)$, and of helium from Panchapakesan and Lumley (1993) 
a)

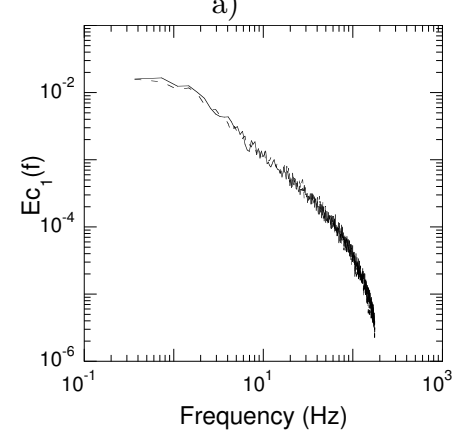

b)

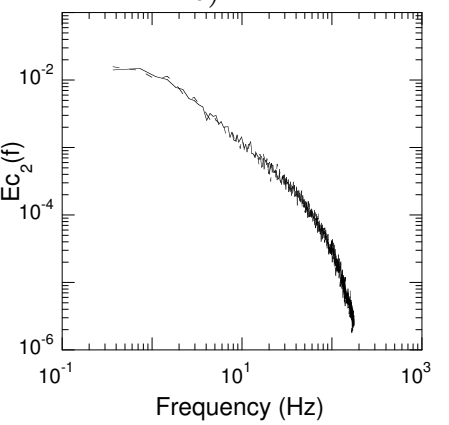

c)

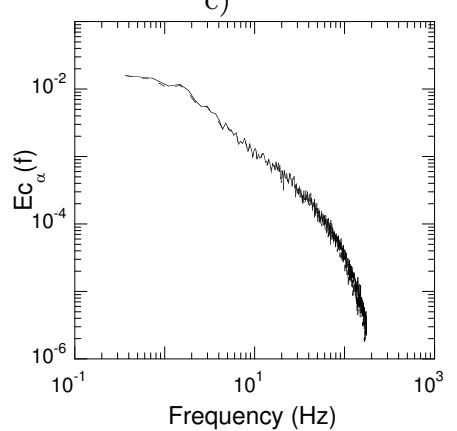

Figure 8. Comparison of the scalar spectra measured in jets with one and both fluorescent dyes $(\mathrm{Re}=10600)$. a) Disodium fluorescein spectra, $E_{c_{1}}(f)$. Disodium fluorescein only (solid line); Both dyes (dashed line). b) Sulforhodamine 101 spectra, $E_{c_{2}}(f)$. Sulforhodamine 101 only (solid line); Both dyes (dashed line). c) Comparison of the two scalar spectra measured in a jet with both fluorescent dyes. $E_{c_{1}}(f)$ (solid line); $E_{c_{2}}(f)$ (dashed line). 
a)

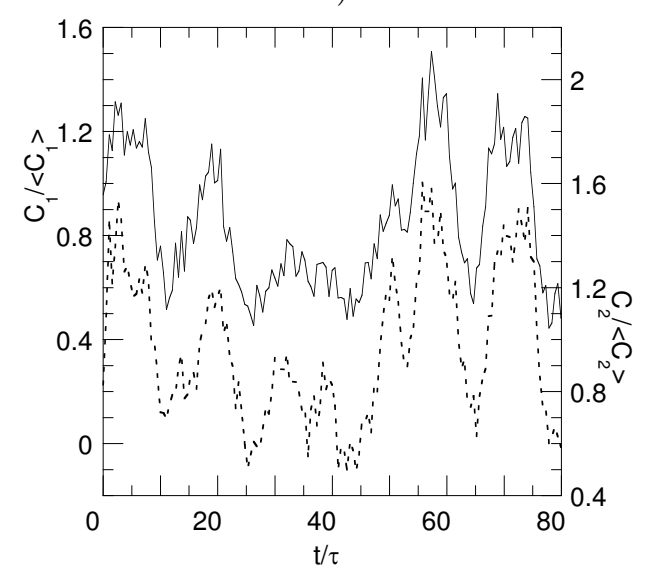

b)

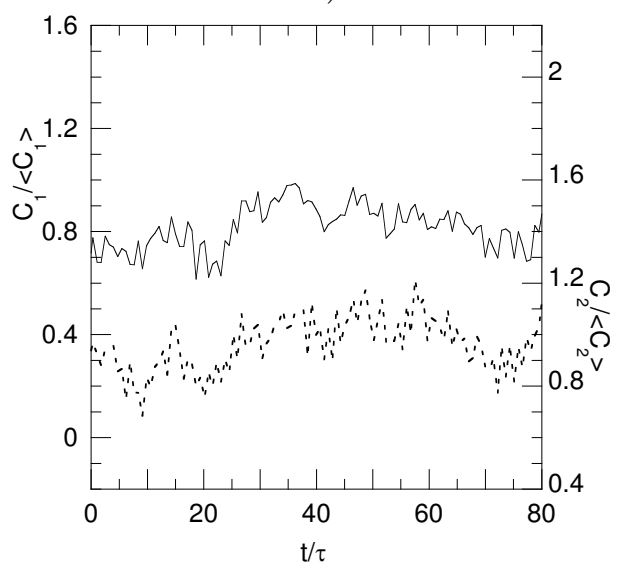

Figure 9. Normalized concentration time series, $C_{1}(t) /\left\langle C_{1}\right\rangle$ (solid line) and $C_{2}(t) /\left\langle C_{2}\right\rangle$ (dashed line) at $\mathrm{r} / \mathrm{d}=0$. (a) $\mathrm{Re}=900$. (b) $\mathrm{Re}=10600$.

a)

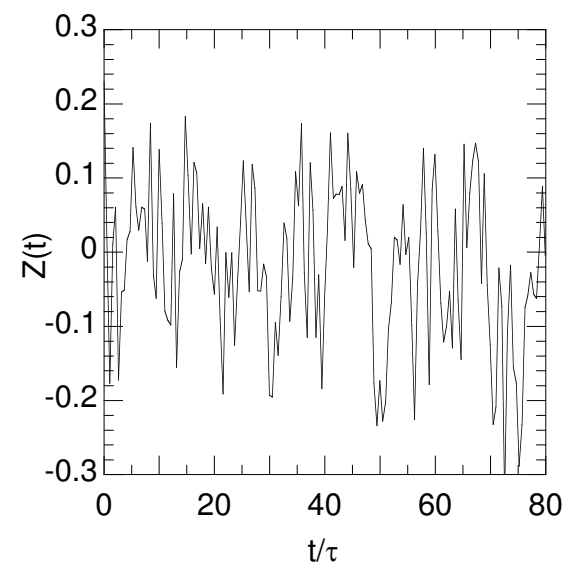

b)

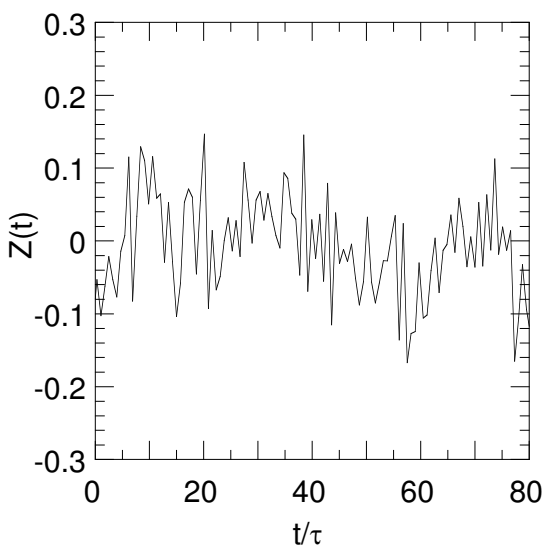

FiguRE 10. Normalized concentration time series, $Z(t)$ at $\mathrm{r} / \mathrm{d}=0$. (a) $\operatorname{Re}=900$. (b) $\operatorname{Re}=$ 10600. 
a)

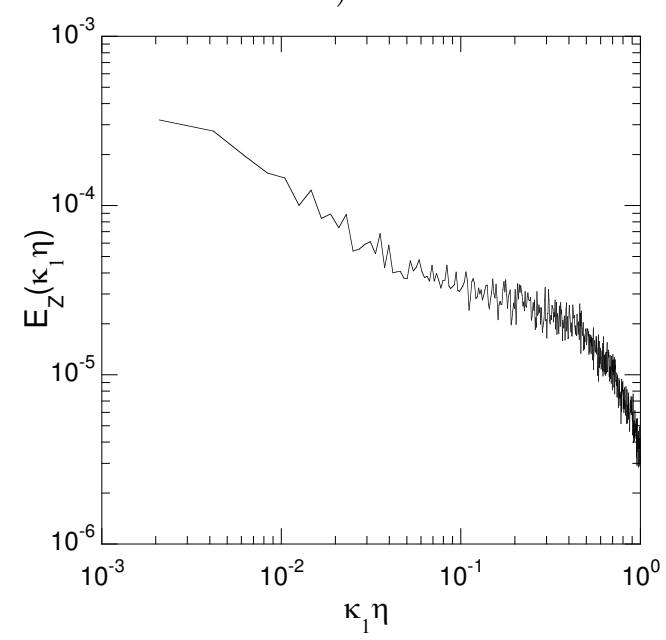

b)

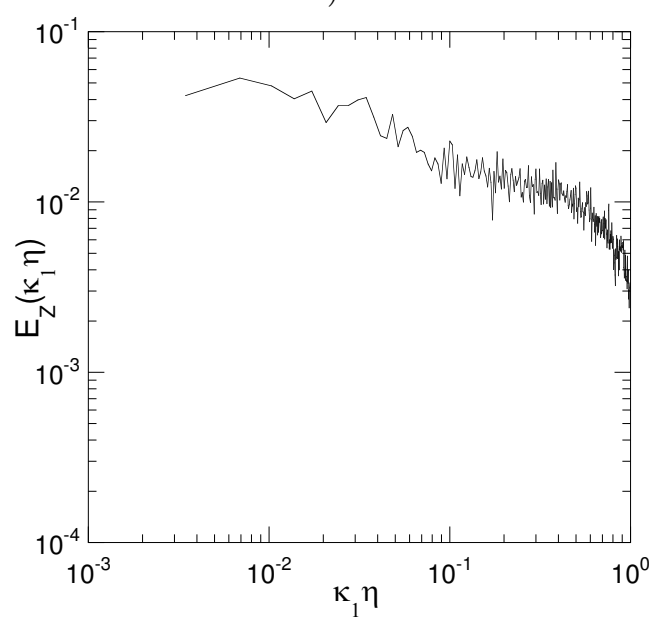

FiguRE 11. Normalized concentration difference spectra, $E_{Z}\left(\kappa_{1} \eta\right)$. a) $\left.R e=10600, r / d=0 . \mathrm{b}\right)$ $R e=900, r / d=6.25$. 


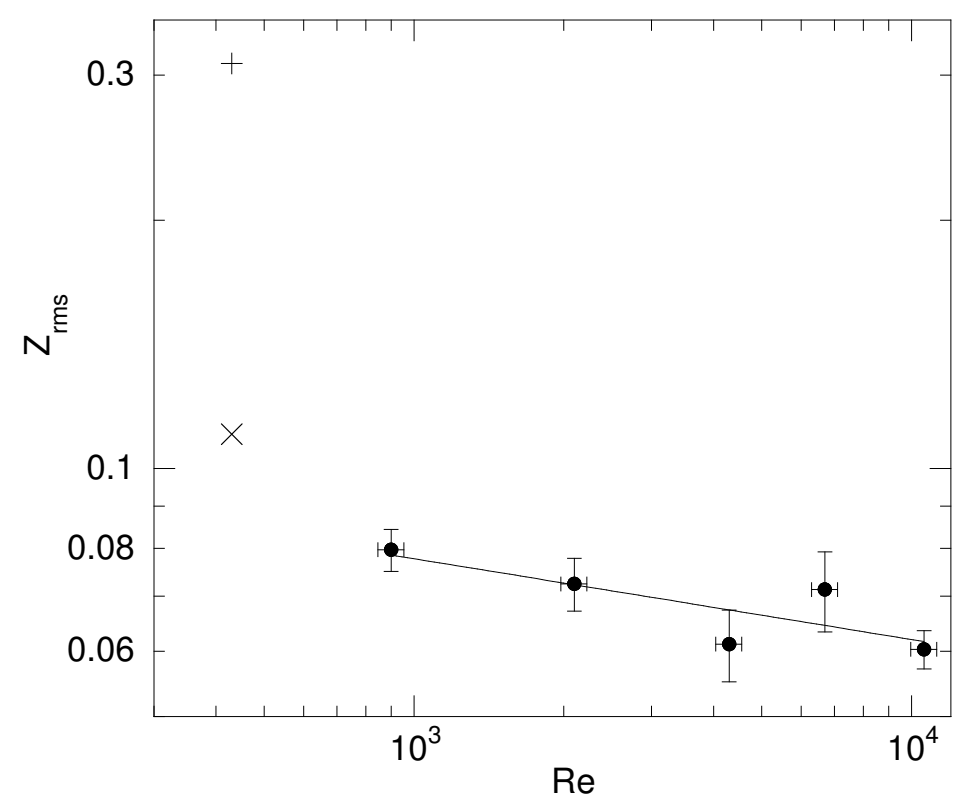

Figure 12. Reynolds number dependence of $Z_{r m s}$ at the jet centreline $(r / d=0)$. The solid line is the best-fit power law to the present data $(\bullet)$, which was measured at $x / d=50$. Error bars are $95 \%$ confidence intervals. The other two symbols are from the experiments of Saylor and Sreenivasan (1998) $-\times: S c_{1}=1200, S c_{2}=4400, x / d=147 ;+: S c_{1}=4400, S c_{2}=77000$, $x / d=147$. 


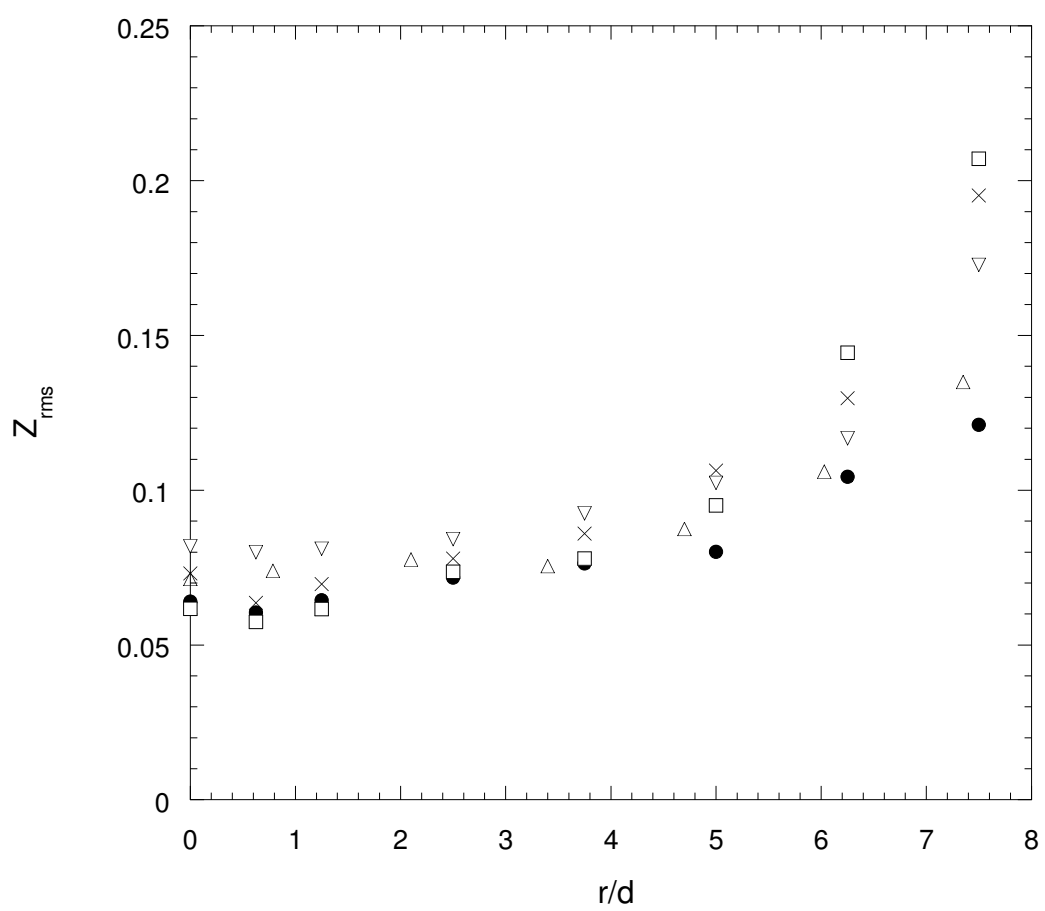

FiguRE 13. Radial distribution of $Z_{r m s}$ at all five Reynolds numbers studied. Re=900: $\nabla$, $\operatorname{Re}=2100: \triangle, \operatorname{Re}=4300: \square, \operatorname{Re}=6700: \times, \operatorname{Re}=10600: \bullet$ 
a)

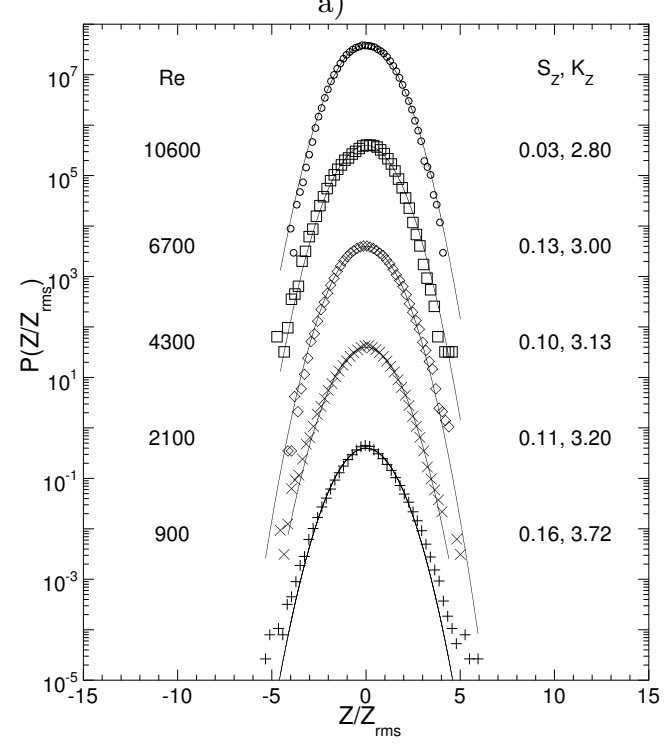

b)

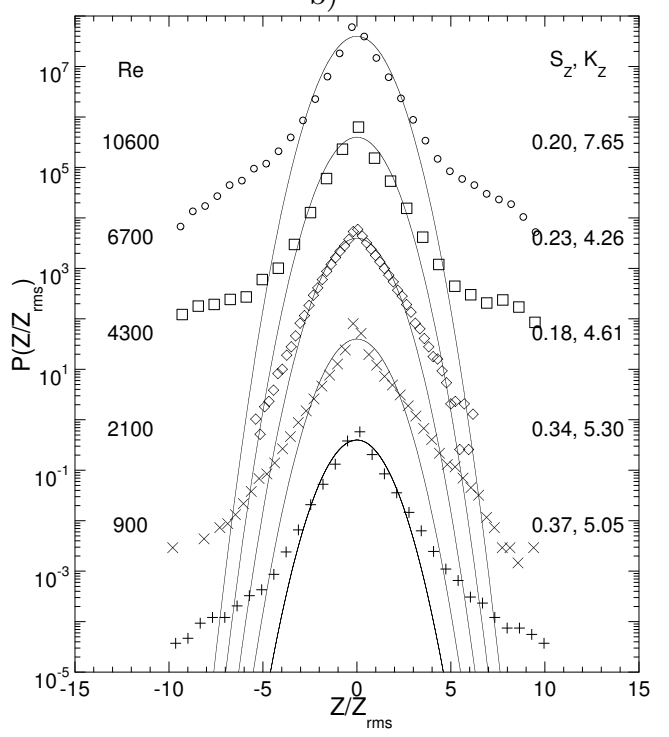

FiguRE 14. PDFs of the normalized concentration difference for $900 \leqslant R e \leqslant 10600$. (a) $r / d=0$ and (b) $r / d=6.25$. The Reynolds number, skewness and kurtosis are labelled on the graphs. Each graph has successively been shifted up by 2 decades with respect to the lower one. The solid lines are standardized normal Gaussian distributions. 


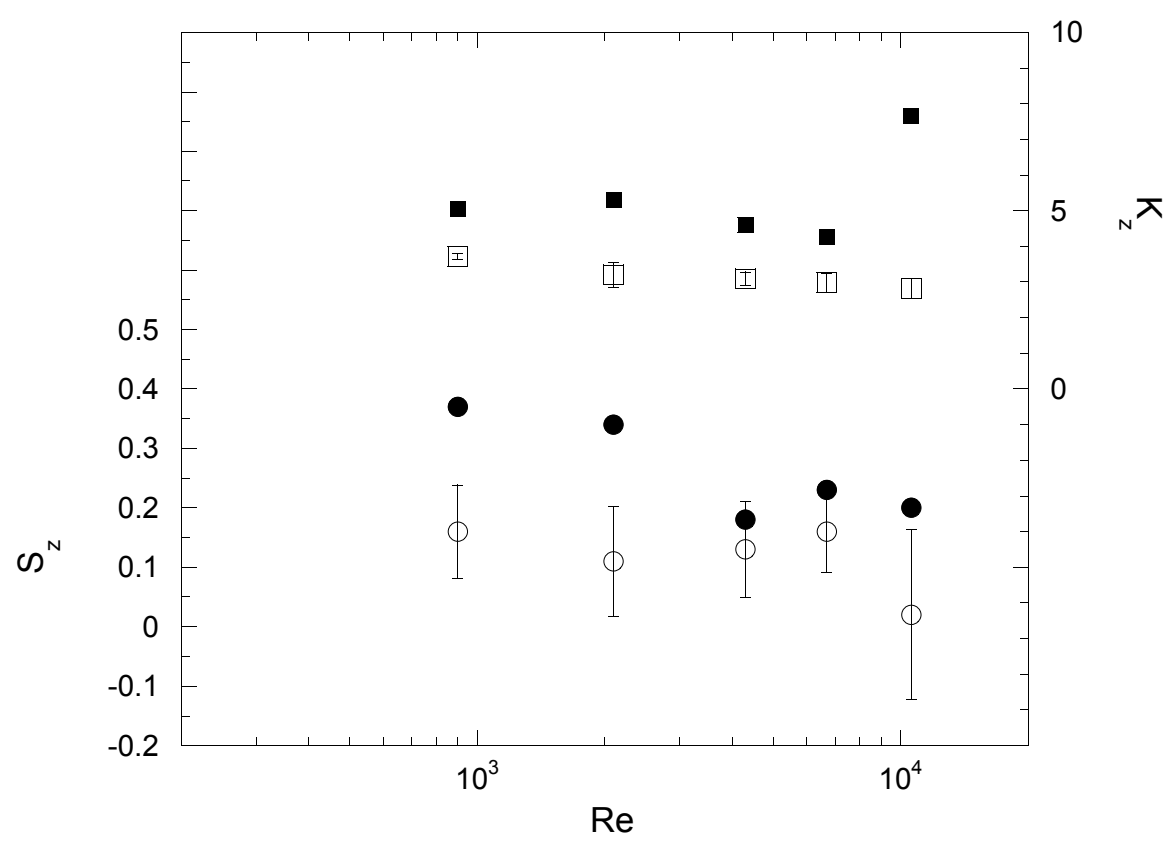

Figure 15. Reynolds number dependence of $S_{Z}$ (circles) and $K_{Z}$ (squares) at $r / d=0$ (open symbols) and $r / d=6.25$ (closed symbols). Error bars are $95 \%$ confidence intervals $(r / d=0$ only). 
a)

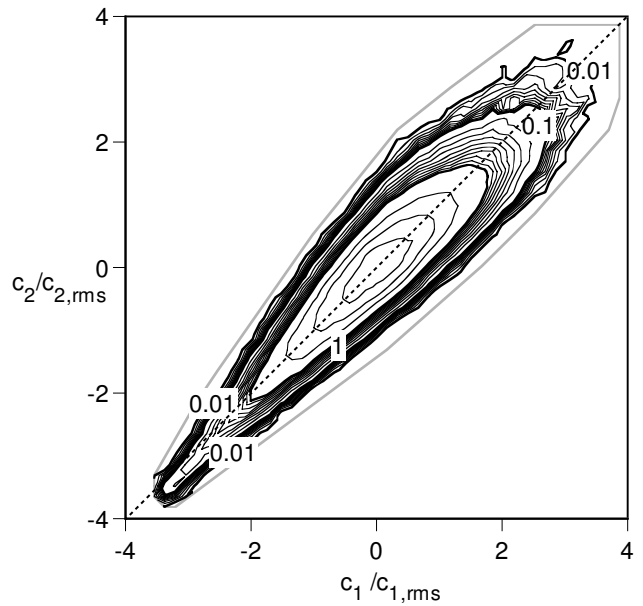

b)

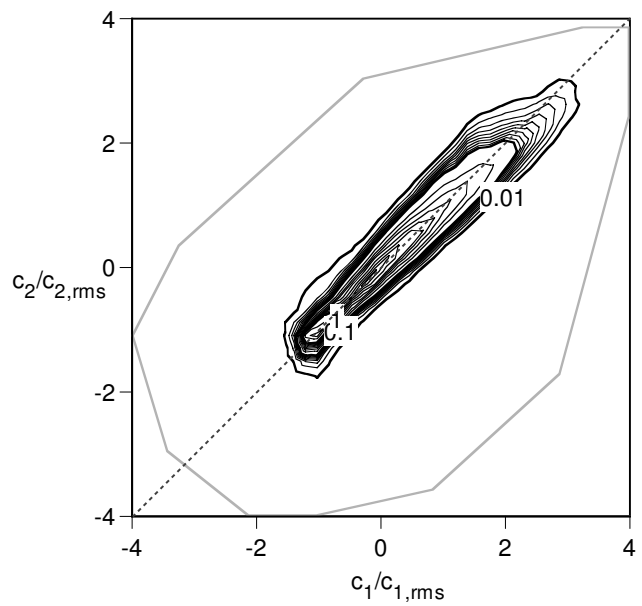

Figure 16. Contour plots of the joint PDFs of disodium fluorescein, $C_{1}$, and sulforhodamine 101, $C_{2}$, at $\operatorname{Re}=10600$. (a) $\mathrm{r} / \mathrm{d}=0$, and (b) $\mathrm{r} / \mathrm{d}=6.25$. 
a)

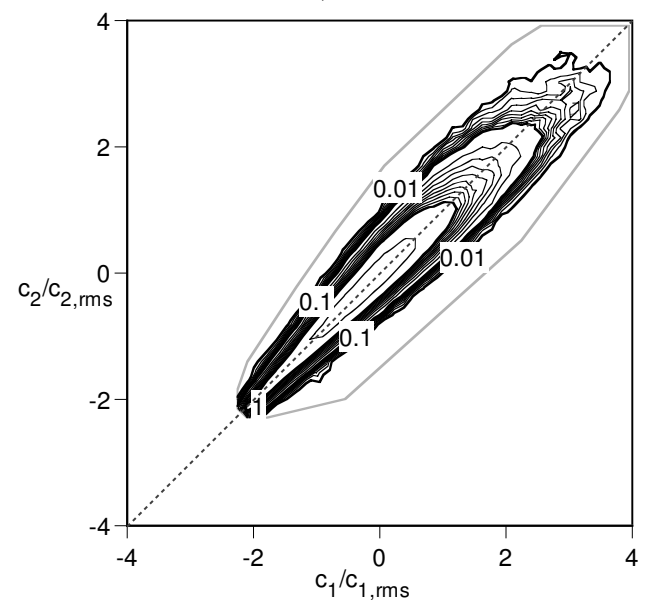

b)

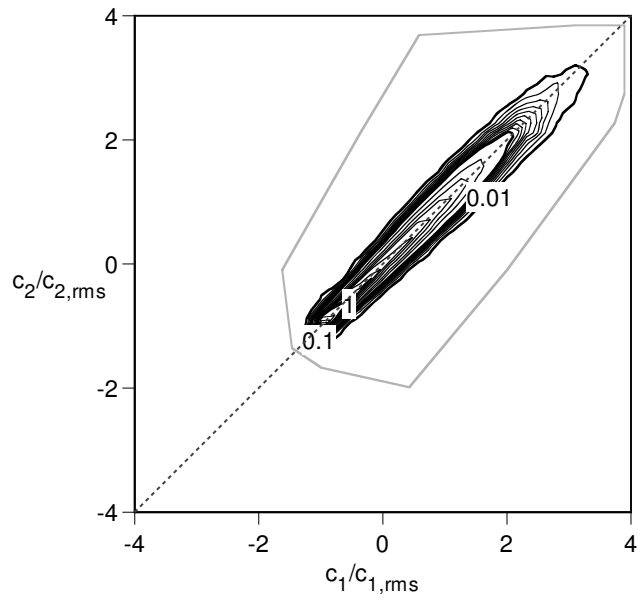

Figure 17. Contour plots of the joint PDFs of disodium fluorescein, $C_{1}$, and sulforhodamine 101, $C_{2}$, at $\operatorname{Re}=900$. (a) $\mathrm{r} / \mathrm{d}=0$ and (b) $\mathrm{r} / \mathrm{d}=6.25$ 
a)

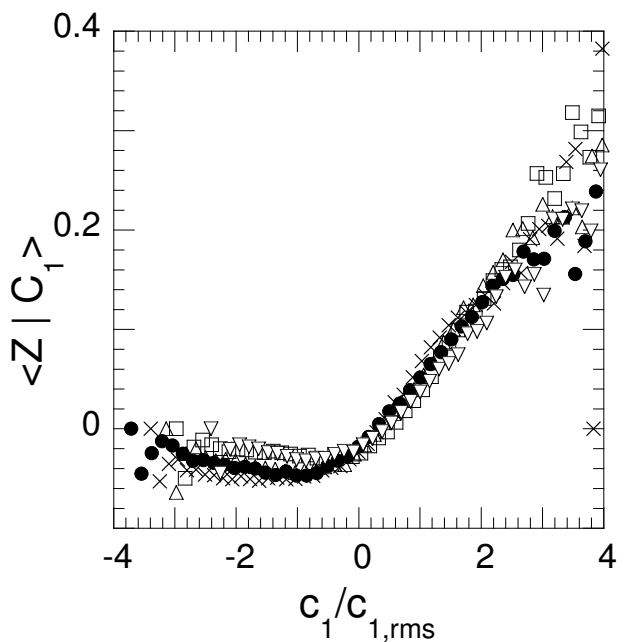

b)

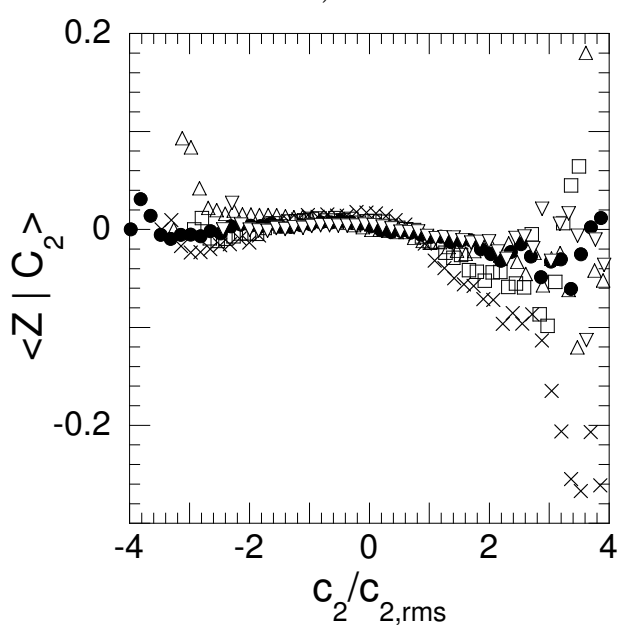

Figure 18. Conditional expectations at $r / d=0$. (a) $\left\langle Z \mid c_{1}\right\rangle$. (b) $\left\langle Z \mid c_{2}\right\rangle$. $\operatorname{Re}=900: \nabla$, $\operatorname{Re}=2100: \triangle, \operatorname{Re}=4300: \square, \operatorname{Re}=6700: \times, \operatorname{Re}=10600: \bullet$. 
a)

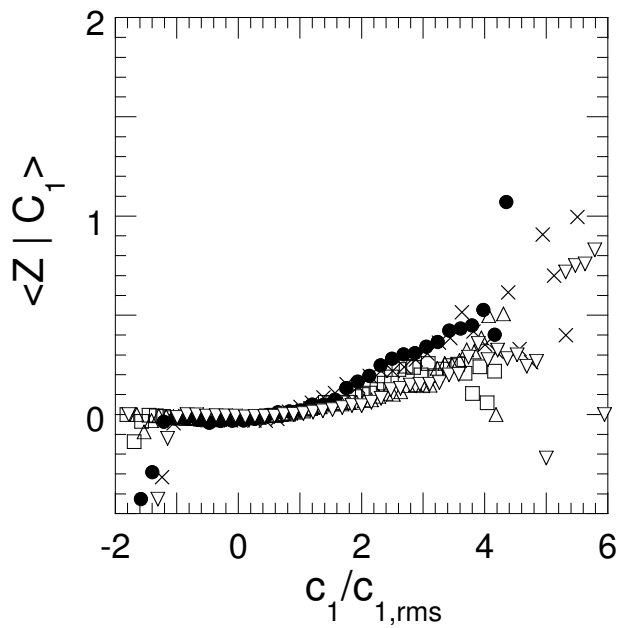

b)

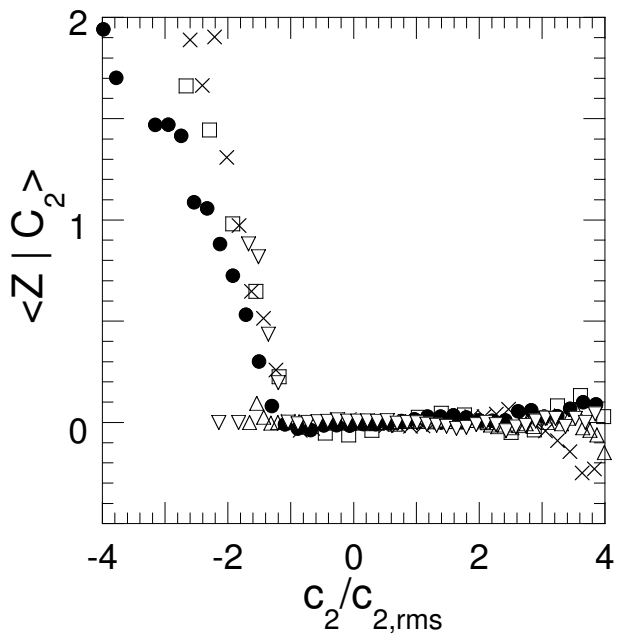

Figure 19. Conditional expectations at $r / d=6.25$. (a) $\left\langle Z \mid c_{1}\right\rangle$. (b) $\left\langle Z \mid c_{2}\right\rangle$. $\operatorname{Re}=900: \nabla$, $\operatorname{Re}=2100: \triangle, \operatorname{Re}=4300: \square, \operatorname{Re}=6700: \times, \operatorname{Re}=10600: \bullet$. 